\title{
Complexity Classifications for Propositional Abduction in Post's Framework*
}

\author{
Nadia Creignou ${ }^{1}$, Johannes Schmidt ${ }^{1}$, Michael Thomas ${ }^{2}$ \\ 1 LIF, UMR CNRS 6166, Aix-Marseille Université \\ 163, Avenue de Luminy, 13288 Marseille Cedex 9, France \\ creignou@lif .univ-mrs.fr \\ johannes.schmidt@lif.univ-mrs.fr \\ 2 Institut für Theoretische Informatik, Gottfried Wilhelm Leibniz Universität \\ Appelstr. 4, 30167 Hannover, Germany \\ thomas@thi . uni-hannover.de
}

\begin{abstract}
In this paper we investigate the complexity of abduction, a fundamental and important form of non-monotonic reasoning. Given a knowledge base explaining the world's behavior it aims at finding an explanation for some observed manifestation. In this paper we consider propositional abduction, where the knowledge base and the manifestation are represented by propositional formulae. The problem of deciding whether there exists an explanation has been shown to be $\Sigma_{2}^{\mathrm{p}}$-complete in general. We focus on formulae in which the allowed connectives are taken from certain sets of Boolean functions. We consider different variants of the abduction problem in restricting both the manifestations and the hypotheses. For all these variants we obtain a complexity classification for all possible sets of Boolean functions. In this way, we identify easier cases, namely NP-complete, coNP-complete and polynomial cases. Thus, we get a detailed picture of the complexity of the propositional abduction problem, hence highlighting sources of intractability. Further, we address the problem of counting the explanations and draw a complete picture for the counting complexity.
\end{abstract}

Keywords: abduction, computational complexity, Post's lattice, propositional logic, boolean connective

\footnotetext{
* Supported by ANR Algorithms and complexity 07-BLAN-0327-04 and DFG grant VO 630/6-1. An earlier version appeared in the Proc. of 12th International Conference on the Principles of Knowledge Representation and Reasoning, KR'2010, Toronto, Canada.
} 


\section{Introduction}

This paper is dedicated to the computational complexity of abduction, a fundamental and important form of non-monotonic reasoning. Given a certain consistent knowledge about the world, abductive reasoning is used to generate explanations (or at least telling if there is one) for observed manifestations. Nowadays abduction has taken on fundamental importance in Artificial Intelligence and has many application areas spanning medical diagnosis [BATJ89], text analysis [HSAM93], system diagnosis [SW01], configuration problems [AFM02], temporal knowledge bases [BL00] and has connections to default reasoning [SL90].

There are several approaches to formalize the problem of abduction. In this paper, we focus on logic based abduction in which the knowledge base is given as a set $\Gamma$ of propositional formulae. We are interested in deciding whether there exists an explanation $E$, i.e., a set of literals consistent with $\Gamma$ such that $\Gamma$ and $E$ together entail the observation.

From a complexity theoretic viewpoint, the abduction problem is very hard since it is $\Sigma_{2}^{\mathrm{p}}$-complete and thus situated at the second level of the polynomial hierarchy [EG95]. This intractability result raises the question for restrictions leading to fragments of lower complexity. Several such restrictions have been considered in previous works. One of the most famous amongst those is Schaefer's framework, where formulae are restricted to generalized conjunctive normal form with clauses from a fixed set of relations [CZ06, NZ05, NZ08].

A similar yet different procedure is to rather require formulae to be constructed from a restricted set of Boolean functions $B$. Such formulae are called $B$-formulae. This approach has first been taken by Lewis, who showed that the satisfiability problem is NP-complete if and only if this set of Boolean functions has the ability to express the negation of implication connective $\not \rightarrow$ [Lew79]. Since then, this approach has been applied to a wide range of problems including equivalence and implication problems [Rei03, BMTV09a], satisfiability and model checking in modal and temporal logics [BHSS06, BSS $\left.{ }^{+} 08\right]$, default logic [BMTV09b], and circumscription [Tho09], among others.

We follow this approach and show that Post's lattice allows to completely classify the complexity of propositional abduction for several variants and all possible sets of allowed Boolean functions. We consider two main variants of the abduction problem. In the first one we may build explanations from positive and negative literals. We refer to this problem as symmetric abduction, ABD for short. The second variant, $\mathrm{P}-\mathrm{ABD}$, is the so-called positive abduction where we allow only positive literals in the explanations.

We first examine the symmetric variant in the case where the representation of the manifestation is a positive literal. We show that depending on the set $B$ of allowed connectives the abduction problem is either $\Sigma_{2}^{\mathrm{p}}$-complete, or NPcomplete, or in $\mathrm{P}$ and $\oplus$ LOGSPACE-hard, or in LogsPaCE. More precisely, we prove that the complexity of this abduction problem is $\Sigma_{2}^{\mathrm{p}}$-complete as soon as $B$ can express one of the functions $x \vee(y \wedge \neg z), x \wedge(y \vee \neg z)$ or $(x \wedge y) \vee(x \wedge \neg z) \vee(y \wedge \neg z)$. It drops to NP-complete when all functions in $B$ are monotonic and have the ability to express one of the functions $x \vee(y \wedge z), x \wedge(y \vee z)$ or $(x \wedge y) \vee(x \wedge z) \vee(y \wedge z)$. 
The problem becomes solvable in polynomial time and is $\oplus$ LOGSPACE-hard if $B$-formulae may depend on more than one variable while being representable as linear equations. Finally the complexity drops to LOGSPACE in all remaining cases. We then complete our study of symmetric abduction with analogous complexity classifications of the variants of $\mathrm{ABD}$ obtained by restricting the manifestation to be respectively a clause, a term or a $B$-formula.

These results are subsequently extended to positive abduction. An overview can be found in Figures 1 and 2.

Please note that in [CZ06], the authors obtained a complexity classification of the abduction problem in the so-called Schaefer's framework. The two classifications are in the same vein since they classify the complexity of abduction for local restrictions on the knowledge base. However the two results are incomparable in the sense that no classification can be deduced from the other. They only overlap in the particular case of the linear connective $\oplus$, for which both types of sets of formulae can be seen as systems of linear equations. This special abduction case has been shown to be decidable in polynomial time in [Zan03].

Besides the decision problem, another natural question is concerned with the number of explanations. This problem refers to the counting problem for abduction. The study of the counting complexity of abduction has been started by Hermann and Pichler ([HP07]). We prove here a trichotomy theorem showing that counting the full explanations of symmetric abduction is either \#-coNPcomplete or \#P-complete or in $\mathrm{FP}$, depending on the set $B$ of allowed connectives. We also consider the counting problem associated with positive abduction, for which we distinguish two frequently used settings: counting either all positive explanations, or counting the subset-minimal. For both formalizations of the counting problem, we get a potentially dichotomous classification with one open case.

The rest of the paper is structured as follows. We first give the necessary preliminaries in Section 2. The abduction problems considered herein are defined in Section 3. In Section 4 we classify the complexity of symmetric abduction, where we first consider the case where the manifestation is a single positive literal (Section 4.1), and then turn to variants in which the manifestations are clauses, terms and restricted formulae (Section 4.2). Section 5 then studies the complexity of positive abduction. An overview of these results is given in Section 6. Finally, Section 7 is dedicated to the counting problem, and Section 8 contains some concluding remarks.

\section{Preliminaries}

Complexity Theory We require standard notions of complexity theory. For the decision problems the arising complexity degrees encompass the classes LOGSPACE, $\mathrm{P}, \mathrm{NP}$, and $\Sigma_{2}^{\mathrm{P}}$. For more background information, the reader is referred to [Pap94]. We furthermore require the class $\oplus$ LOGSPACE defined as the class of languages $L$ such that there exists a nondeterministic logspace Turing machine that exhibits an odd number of accepting paths if and only if $x \in L$, for all $x$ [BDHM92]. 
It holds that LOGSPACE $\subseteq \oplus$ LOGSPACE $\subseteq$ P. For our hardness results we employ logspace many-one reductions, defined as follows: a language $A$ is logspace many-one reducible to some language $B$ (written $A \leq_{\mathrm{m}}^{\log } B$ ) if there exists a logspace-computable function $f$ such that $x \in A$ if and only if $f(x) \in B$.

A counting problem is represented using a witness function $w$, which for every input $x$ returns a finite set of witnesses. This witness function gives rise to the following counting problem: given an instance $x$, find the cardinality $|w(x)|$ of the witness set $w(x)$. The class \#P is the class of counting problems naturally associated with decision problems in NP. According to [HV95] if $\mathcal{C}$ is a complexity class of decision problems, we define \#.C to be the class of all counting problems whose witness function is such that the size of every witness $y$ of $x$ is polynomially bounded in the size of $x$, and checking whether $y \in w(x)$ is in $\mathcal{C}$. Thus, we have $\# \mathrm{P}=\# \cdot \mathrm{P}$ and $\# \mathrm{P} \subseteq \# \cdot$ coNP. Completeness of counting problems is usually proved by means of Turing reductions. A stronger notion is the parsimonious reduction where the exact number of solutions is conserved by the reduction function.

Propositional formulae We assume familiarity with propositional logic. The set of all propositional formulae is denoted by $\mathcal{L}$. A model for a formula $\varphi$ is a truth assignment to the set of its variables that satisfies $\varphi$. Further we denote by $\varphi[\alpha / \beta]$ the formula obtained from $\varphi$ by replacing all occurrences of $\alpha$ with $\beta$. For a given set $\Gamma$ of formulae, we write $\operatorname{Vars}(\Gamma)$ to denote the set of variables occurring in $\Gamma$. We identify finite $\Gamma$ with the conjunction of all the formulae in $\Gamma$, $\bigwedge_{\varphi \in \Gamma} \varphi$. Naturally, $\Gamma[\alpha / \beta]$ then stands for $\bigwedge_{\varphi \in \Gamma} \varphi[\alpha / \beta]$. For any formula $\varphi \in \mathcal{L}$, we write $\Gamma \models \varphi$ if $\Gamma$ entails $\varphi$, i.e., if every model of $\Gamma$ also satisfies $\varphi$.

A literal $l$ is a variable $x$ or its negation $\neg x$. Given a set of variables $V, \operatorname{Lits}(V)$ denotes the set of all literals formed upon the variables in $V$, i.e., $\operatorname{Lits}(V):=$ $V \cup\{\neg x \mid x \in V\}$. A clause is a disjunction of literals and a term is a conjunction of literals.

Clones of Boolean Functions A clone is a set of Boolean functions that is closed under superposition, i.e., it contains all projections (that is, the functions $f\left(a_{1}, \ldots, a_{n}\right)=a_{k}$ for all $1 \leq k \leq n$ and $\left.n \in \mathbb{N}\right)$ and is closed under arbitrary composition. Let $B$ be a finite set of Boolean functions. We denote by $[B]$ the smallest clone containing $B$ and call $B$ a base for $[B]$. In 1941 Post identified the set of all clones of Boolean functions [Pos41]. He gave a finite base for each of the clones and showed that they form a lattice under the usual $\subseteq$-relation, hence the name Post's lattice (see, e.g., Figure 1). To define the clones we introduce the following notions, where $f$ is an $n$-ary Boolean function:

- $f$ is c-reproducing if $f(c, \ldots, c)=c, c \in\{0,1\}$.

- $f$ is monotonic if $a_{1} \leq b_{1}, \ldots, a_{n} \leq b_{n}$ implies $f\left(a_{1}, \ldots, a_{n}\right) \leq f\left(b_{1}, \ldots, b_{n}\right)$.

- $f$ is c-separating of degree $k$ if for all $A \subseteq f^{-1}(c)$ of size $|A|=k$ there exists an $i \in\{1, \ldots, n\}$ such that $\left(a_{1}, \ldots, a_{n}\right) \in A$ implies $a_{i}=c, c \in\{0,1\}$.

- $f$ is c-separating if $f$ is $c$-separating of degree $\left|f^{-1}(c)\right|$.

$-f$ is self-dual if $f \equiv \operatorname{dual}(f)$, where $\operatorname{dual}(f)\left(x_{1}, \ldots, x_{n}\right):=\neg f\left(\neg x_{1}, \ldots, \neg x_{n}\right)$. 
- $f$ is affine if $f \equiv x_{1} \oplus \cdots \oplus x_{n} \oplus c$ with $c \in\{0,1\}$.

A list of all clones with definitions and finite bases is given in Table 1 on page 24. A propositional formula using only functions from $B$ as connectives is called a $B$-formula. The set of all $B$-formulae is denoted by $\mathcal{L}(B)$.

Let $f$ be an $n$-ary Boolean function. A $B$-formula $\varphi$ such that $\operatorname{Vars}(\varphi)=$ $\left\{x_{1}, \ldots, x_{n}, y_{1}, \ldots, y_{m}\right\}$ is a $B$-representation of $f$ if for all $a_{1}, \ldots, a_{n}, b_{1}, \ldots, b_{m} \in$ $\{0,1\}$ it holds that $f\left(a_{1}, \ldots, a_{n}\right)=1$ if and only if every $\sigma: \operatorname{Vars}(\varphi) \longrightarrow$ $\{0,1\}$ with $\sigma\left(x_{i}\right)=a_{i}$ and $\sigma\left(y_{i}\right)=b_{i}$ for all relevant $i$ satisfies $\varphi$. Such a $B$-representation exists for every $f \in[B]$. Yet, it may happen that the $B$ representation of some function uses some input variable more than once.

Example 2.1. Let $h(x, y)=x \wedge \neg y$. An $\{h\}$-representation of the function $x \wedge y$ is $h(x, h(x, y))$.

\section{The Abduction Problem}

Let $B$ be a finite set of Boolean functions. We are interested in the propositional abduction problem parameterized by the set $B$ of allowed connectives. We define the abduction problem for $B$-formulae as

$$
\begin{array}{ll}
\text { Problem: } & \operatorname{ABD}(B) \\
\text { Instance: } & \mathcal{P}=(\Gamma, A, \varphi), \text { where } \\
& -\Gamma \subseteq \mathcal{L}(B) \text { is a set of } B \text {-formulae, } \\
& -A \subseteq \operatorname{Vars}(\Gamma) \text { is a set of variables, } \\
& -\varphi \in \mathcal{L} \text { is a formula with } \operatorname{Vars}(\varphi) \subseteq \operatorname{Vars}(\Gamma) \backslash A .
\end{array}
$$

Question: Is there a set $E \subseteq \operatorname{Lits}(A)$ such that $\Gamma \wedge E$ is satisfiable and $\Gamma \wedge E \models \varphi$ (or equivalently $\Gamma \wedge E \wedge \neg \varphi$ is unsatisfiable)?

The set $\Gamma$ represents the knowledge base. The set $A$ is called the set of hypotheses and $\varphi$ is called manifestation or query. Furthermore, if such a set $E$ exists, it is called an explanation or a solution of the abduction problem. It is called a full explanation if $\operatorname{Vars}(E)=A$. Observe that every explanation can be extended to a full one. We will consider several restrictions of the manifestations of this problem. To indicate them, we introduce a second argument $\mathcal{M}$ meaning that $\varphi$ is required to be

- Q (resp. PQ, NQ): a single literal (resp. positive literal, negative literal),

- C (resp. PC, NC): a clause (resp. positive clause, negative clause),

- T (resp. PT, NT): a term (resp. positive term, negative term),

$-\mathcal{L}(B)$ : a $B$-formula.

We refer to the above defined abduction problem as symmetric abduction, since every variable of the hypotheses $A$ may be taken positive or negative to construct an explanation. We will also consider positive abduction, where we are interested in purely positive explanations only. To indicate this, we add the prefix "P-". 
Thus, for an instance $(\Gamma, A, \varphi)$ of $\mathrm{P}-\mathrm{ABD}(B, \mathcal{M})$, every solution $E$ of $(\Gamma, A, \varphi)$ has to satisfy $E \subseteq A$.

The following important lemma makes clear the role of the constants in our abduction problem. It often reduces the number of cases to be considered.

Lemma 3.1. Let $B$ be a finite set of Boolean functions.

1. If $\mathcal{M} \in\{\mathrm{Q}, \mathrm{C}, \mathrm{T}, \mathcal{L}(B)\}$, then

$$
\begin{aligned}
\operatorname{ABD}(B, \mathcal{M}) & \equiv_{\mathrm{m}}^{\log } \operatorname{ABD}(B \cup\{1\}, \mathcal{M}), \\
\mathrm{P}-\operatorname{ABD}(B, \mathcal{M}) & \equiv_{\mathrm{m}}^{\log } \mathrm{P}-\mathrm{ABD}(B \cup\{1\}, \mathcal{M}) .
\end{aligned}
$$

2. If $\mathcal{M} \in\{\mathrm{Q}, \mathrm{C}, \mathrm{T}\}$ and $\vee \in[B]$, then

$$
\operatorname{ABD}(B, \mathcal{M}) \equiv{ }_{\mathrm{m}}^{\log } \operatorname{ABD}(B \cup\{0\}, \mathcal{M}) .
$$

Proof. To reduce $\operatorname{ABD}(B \cup\{1\}, \mathcal{M})$ to $\operatorname{ABD}(B, \mathcal{M})$ we transform any instance of the first problem in replacing every occurrence of 1 by a fresh variable $t$ and adding the unit clause $(t)$ to the knowledge base. The same reduction works for P-ABD. To prove $\operatorname{ABd}(B \cup\{0\}, \mathcal{M}) \equiv_{\mathrm{m}}^{\log } \operatorname{ABD}(B, \mathcal{M})$, let $\mathcal{P}=(\Gamma, A, \psi)$ be an instance of the first problem and $f$ be a fresh variable. Since $\mathcal{M} \in\{\mathrm{Q}, \mathrm{C}, \mathrm{T}\}$, we can suppose w.l.o.g. that $\psi$ does not contain 0 . We map $\mathcal{P}$ to $\mathcal{P}^{\prime}=\left(\Gamma^{\prime}, A^{\prime}, \psi\right)$, where $\Gamma^{\prime}$ is the $B$-representation of $\{\varphi[0 / f] \vee f \mid \varphi \in \Gamma\}$ and $A^{\prime}=A \cup\{f\}$.

Of course this lemma holds also for purely positive/negative queries, clauses or terms, i.e., Q, C, T can be replaced by $\mathrm{PQ}, \mathrm{PC}, \mathrm{PT}$ or $\mathrm{NQ}, \mathrm{NC}, \mathrm{NT}$, respectively.

Observe that if $B_{1}$ and $B_{2}$ are two sets of Boolean functions such that $B_{1} \subseteq$ $\left[B_{2}\right]$, then every function of $B_{1}$ can be expressed by a $B_{2}$-formula, namely by its $B_{2}$-representation. This way there is a canonical reduction between $\operatorname{ABD}\left(B_{1}, \mathcal{M}\right)$ and $\operatorname{ABD}\left(B_{2}, \mathcal{M}\right)$ if $B_{1} \subseteq\left[B_{2}\right]$ : replace all $B_{1}$-connectives by their $B_{2}$-representation. Note that this reduction is not necessarily polynomial: Since the $B_{2}$-representation of some function may use some input variable more than once, the formula size may grow exponentially. Nevertheless we will use this reduction very frequently, avoiding an exponential blow-up by special structures of the $B_{1}$-formulae.

\section{The complexity of $\operatorname{Abd}(B)$}

We commence with the symmetric abduction problem $\operatorname{ABD}(B)$ The results of this section are summarized in Figure 1. We will first focus on the case where $\varphi$ is a single positive literal, thus discussing the problem $\operatorname{ABD}(B, \mathrm{PQ})$.

\subsection{The complexity of $\operatorname{Abd}(B, P Q)$}

Theorem 4.1. Let $B$ be a finite set of Boolean functions. Then the symmetric abduction problem for propositional B-formulae with a positive literal manifestation, $\operatorname{ABD}(B, \mathrm{PQ})$, is 
1. $\Sigma_{2}^{\mathrm{p}}$-complete if $\mathrm{S}_{02} \subseteq[B]$ or $\mathrm{S}_{12} \subseteq[B]$ or $\mathrm{D}_{1} \subseteq[B]$,

2. NP-complete if $\mathrm{S}_{00} \subseteq[B] \subseteq \mathrm{M}$ or $\mathrm{S}_{10} \subseteq[B] \subseteq \mathrm{M}$ or $\mathrm{D}_{2} \subseteq[B] \subseteq \mathrm{M}$,

3. in $\mathrm{P}$ and $\oplus$ LOGSPACE-hard if $\mathrm{L}_{2} \subseteq[B] \subseteq \mathrm{L}$, and

4. in LOGSPACE in all other cases.

Remark 4.2. For such a classification a natural question is: given $B$, how hard is it to determine the complexity of $\operatorname{ABD}(B, \mathrm{PQ})$ ? Solving this task requires checking whether certain clones are included in $[B]$ (for lower bounds) and whether $B$ itself is included in certain clones (for upper bounds). As shown in [Vol09], the complexity of checking whether certain Boolean functions are included in a clone depends on the representation of the Boolean functions. If all functions are given by their truth table then the problem is in quasi-polynomial-size $\mathrm{AC}^{0}$, while if the input functions are given in a compact way, i.e., by circuits, then the above problem becomes coNP-complete.

We split the proof of Theorem 4.1 into several propositions.

Proposition 4.3. Let $B$ be a finite set of Boolean functions such that $[B] \subseteq \mathrm{E}$ or $[B] \subseteq \mathrm{N}$ or $[B] \subseteq \mathrm{V}$. Then $\mathrm{ABD}(B, \mathrm{PQ}) \in$ LOGSPACE.

Proof. Let $\mathcal{P}=(\Gamma, A, q)$ be an instance of $\operatorname{ABD}(B, \mathrm{PQ})$.

For $[B]=\mathrm{N}$ or $\mathrm{E}, \Gamma$ is equivalent to a set of literals, hence $\mathcal{P}$ has the empty set as a solution if $\mathcal{P}$ possesses a solution at all. Finally notice that satisfiability of a set of $\mathrm{N}$-formulae can be tested in logarithmic space [Sch05].

For $[B]=\mathrm{V}$ each formula $\varphi \in \Gamma$ is equivalent to either a constant or disjunction. It holds that $(\Gamma, A, q)$ has a solution if and only if $\Gamma$ contains a formula $\varphi \equiv q \vee x_{1} \vee \cdots \vee x_{k}$ such that $\left\{x_{1}, \ldots x_{k}\right\} \subseteq A$, and $\Gamma\left[x_{1} / 0, \ldots, x_{k} / 0\right]$ is satisfiable. This can be tested in logarithmic space, as substitution of symbols and evaluation of $\mathrm{V}$-formulae can all be performed in logarithmic space.

Proposition 4.4. Let $B$ be a finite set of Boolean functions such that $\mathrm{L}_{2} \subseteq$ $[B] \subseteq \mathrm{L}$. Then $\operatorname{ABD}(B, \mathrm{PQ})$ is $\oplus$ LOGSPACE-hard and contained in $\mathrm{P}$.

Proof. In this case, deciding whether an instance of $\operatorname{ABD}(B, \mathrm{PQ})$ has a solution logspace reduces to the problem of deciding whether a propositional abduction problem in which the knowledge base is a set of linear equations has a solution. This has been shown to be decidable in polynomial time in [Zan03].

As for the $\oplus$ LogspaCE-hardness, let $B$ be such that $[B]=\mathrm{L}_{2}$. Consider the $\oplus$ LOGSPACE-complete problem to determine whether a system of linear equations $S$ over $G F(2)$ has a solution [BDHM92]. Note that $\oplus$ LOGSPACE is closed under complement, so deciding whether such a system has no solution is also $\oplus$ LOGSPACE-complete. Let $S=\left\{s_{1}, \ldots, s_{m}\right\}$ be such a system of linear equations over variables $\left\{x_{1}, \ldots, x_{n}\right\}$. Then, for all $1 \leq i \leq m$, the equation $s_{i}$ is of the form $x_{i_{1}}+\cdots+x_{i_{n_{i}}}=c_{i}(\bmod 2)$ with $c_{i} \in\{0,1\}$ and $i_{1}, \ldots, i_{n_{i}} \in\{1, \ldots, n\}$. We map $S$ to a set of affine formulae $\Gamma=\left\{\varphi_{1}, \ldots, \varphi_{m}\right\}$ over variables $\left\{x_{1}, \ldots, x_{n}, q\right\}$ via

$$
\begin{array}{ll}
\varphi_{i}:=x_{i_{1}} \oplus \cdots \oplus x_{i_{n_{i}}} \oplus 1 & \text { if } c_{i}=0 \text { and } \\
\varphi_{i}:=x_{i_{1}} \oplus \cdots \oplus x_{i_{n_{i}}} & \text { if } c_{i}=1 .
\end{array}
$$


Now define

$$
\begin{aligned}
\Gamma^{\prime}: & =\left\{\varphi_{i} \oplus q \mid \varphi_{i} \in \Gamma \text { is such that } \varphi_{i}(1, \ldots, 1)=0\right\} \\
& \cup\left\{\varphi_{i} \mid \varphi_{i} \in \Gamma \text { is such that } \varphi_{i}(1, \ldots, 1)=1\right\} .
\end{aligned}
$$

$\Gamma^{\prime}$ is obviously satisfied by the assignment mapping all propositions to 1 . It furthermore holds that $S$ has no solution if and only if $\Gamma^{\prime} \wedge \neg q$ is unsatisfiable. Hence, we obtain that $S$ has no solution if and only if the propositional abduction problem $\left(\Gamma^{\prime}, \emptyset, q\right)$ has an explanation.

It remains to transform $\Gamma^{\prime}$ into a set of $B$-formulae in logarithmic space. Since $[B]=\mathrm{L}_{2}$, we have $x \oplus y \oplus z \in[B]$. We insert parentheses in every formula $\varphi$ of $\Gamma^{\prime}$ in such a way that we get a ternary $\oplus$-tree of logarithmic depth whose leaves are either a proposition or the constant 1 . Then we replace every node $\oplus$ by its equivalent $B$-formula. Thus we get a $(B \cup\{1\})$-formula of size polynomial in the size of the original one. Lemma 3.1 allows to conclude.

Observe that in the cases $[B] \subseteq \mathrm{L},[B] \subseteq \mathrm{E}$, and $[B] \subseteq \mathrm{V}$, the abduction problem for $B$-formulae is self-reducible. Roughly speaking, this means that given an instance $\mathcal{P}$ and a literal $l$, we can efficiently compute an instance $\mathcal{P}^{\prime}$ such that the question whether there exists an explanation $E$ with $l \in E$ reduces to the question whether $\mathcal{P}^{\prime}$ admits solutions. It is well-known that for self-reducible problems whose decision problem is in $\mathrm{P}$, the lexicographic first solution can be computed in FP. It is an easy exercise to extend this algorithm to enumerate all solutions in lexicographic order with polynomial delay and polynomial space. Thus, if $[B] \subseteq \mathrm{L}$ or $[B] \subseteq \mathrm{E}$ or $[B] \subseteq \mathrm{V}$, the explanations of $\operatorname{ABD}(B, \mathrm{PQ})$ can be enumerated with polynomial delay and polynomial space according to Proposition 4.3 and 4.4 .

Proposition 4.5. Let $B$ be a finite set of Boolean functions such that $\mathrm{S}_{00} \subseteq$ $[B] \subseteq \mathrm{M}$ or $\mathrm{S}_{10} \subseteq[B] \subseteq \mathrm{M}$ or $\mathrm{D}_{2} \subseteq[B] \subseteq \mathrm{M}$. Then $\mathrm{ABD}(B, \mathrm{PQ})$ is NP-complete.

Proof. We first show that $\operatorname{ABd}(B, \mathrm{PQ})$ is efficiently verifiable. Let $\mathcal{P}=(\Gamma, A, q)$ be an $\operatorname{ABD}(B, \mathrm{PQ})$-instance and $E \subseteq \operatorname{Lits}(A)$ be a candidate for an explanation. Define $\Gamma^{\prime}$ as the set of formulae obtained from $\Gamma$ by replacing each occurrence of the proposition $x$ with 0 if $\neg x \in E$, and each occurrence of the proposition $x$ with 1 if $x \in E$. It holds that $E$ is a solution for $\mathcal{P}$ if $\Gamma^{\prime}$ is satisfiable and $\Gamma^{\prime}[q / 0]$ is not. These tests can be performed in polynomial time, because $\Gamma^{\prime}$ is a set of monotonic formulae [Lew79]. Hence, $\operatorname{ABD}(B, \mathrm{PQ}) \in \mathrm{NP}$.

Next we give a reduction from the NP-complete problem 2-IN-3-SAT, i.e., the problem to decide whether there exists an assignment that satisfies exactly two propositions in each clause of a given formula in conjunctive normal form with exactly three positive propositions per clause, see [Sch78]. Let $\varphi:=\bigwedge_{i \in I} c_{i}$ with $c_{i}=x_{i 1} \vee x_{i 2} \vee x_{i 3}, i \in I$, be the given formula. We map $\varphi$ to the following instance $\mathcal{P}=(\Gamma, A, q)$. Let $q_{i}, i \in I$, be fresh, pairwise distinct propositions and 
let $A:=\operatorname{Vars}(\varphi) \cup\left\{q_{i} \mid i \in I\right\}$. The set $\Gamma$ is defined as

$$
\begin{aligned}
\Gamma: & =\left\{c_{i} \mid i \in I\right\} \\
& \cup\left\{x_{i 1} \vee x_{i 2} \vee q_{i}, x_{i 1} \vee x_{i 3} \vee q_{i}, x_{i 2} \vee x_{i 3} \vee q_{i} \mid i \in I\right\} \\
& \cup\left\{\bigvee_{i \in I} \bigwedge_{j=1}^{3} x_{i j} \vee \bigvee_{i \in I} q_{i} \vee q\right\} .
\end{aligned}
$$

We show that there is an assignment that sets to true exactly two propositions in each clause of $\varphi$ if and only if $\mathcal{P}$ has a solution. First, suppose that there exists an assignment $\sigma$ such that for all $i \in I$, there is a permutation $\pi_{i}$ of $\{1,2,3\}$ such that $\sigma\left(x_{i \pi_{i}(1)}\right)=0$ and $\sigma\left(x_{i \pi_{i}(2)}\right)=\sigma\left(x_{i \pi_{i}(3)}\right)=1$. Thus (1) and (2) are satisfied, and (3) is equivalent to $\bigvee_{i \in I} q_{i} \vee q$. From this, it is readily observed that $\{\neg x \mid \sigma(x)=0\} \cup\left\{\neg q_{i} \mid i \in I\right\}$ is a solution to $\mathcal{P}$.

Conversely, suppose that $\mathcal{P}$ has an explanation $E$ that is w.l.o.g. full. Then $\Gamma \wedge E$ is satisfiable and $\Gamma \wedge E \models q$. Let $\sigma$ : $\operatorname{Vars}(\Gamma) \longrightarrow\{0,1\}$ be an assignment that satisfies $\Gamma \wedge E$. Then, for any $x \in A, \sigma(x)=0$ if $\neg x \in E$, and $\sigma(x)=1$ otherwise. Since $\Gamma \wedge E$ entails $q$ and as the only occurrence of $q$ is in (3), we obtain that $\sigma$ sets to 0 each $q_{i}$ and at least one proposition in each clause of $\varphi$. Consequently, from (2) it follows that $\sigma$ sets to 1 at least two propositions in each clause of $\varphi$. Therefore, $\sigma$ sets to 1 exactly two propositions in each clause of $\varphi$.

It remains to show that $\mathcal{P}$ can be transformed into an $\mathrm{ABD}(B, \mathrm{PQ})$-instance for all considered $B$. Observe that $\vee \in[B \cup\{1\}]$ and $\left[\mathrm{S}_{00} \cup\{0,1\}\right]=\left[\mathrm{D}_{2} \cup\{0,1\}\right]=$ $\left[\mathrm{S}_{10} \cup\{0,1\}\right]=\mathrm{M}$. Therefore due to Lemma 3.1 it suffices to consider the case $[B]=\mathrm{M}$. Using the associativity of $\vee$ rewrite (3) as an $\vee$-tree of logarithmic depth and replace all the connectives in $\Gamma$ by their B-representation $(\vee, \wedge \in[B])$.

Proposition 4.6. Let $B$ be a finite set of Boolean functions such that $\mathrm{S}_{02} \subseteq[B]$ or $\mathrm{S}_{12} \subseteq[B]$ or $\mathrm{D}_{1} \subseteq[B]$. Then $\mathrm{ABD}(B, \mathrm{PQ})$ is $\Sigma_{2}^{\mathrm{p}}$-complete.

Proof. Membership in $\Sigma_{2}^{\mathrm{p}}$ is easily seen to hold: given an instance $(\Gamma, A, q)$, guess an explanation $E$ and subsequently verify that $\Gamma \wedge E$ is satisfiable and $\Gamma \wedge E \wedge \neg q$ is not.

Observe that $\vee \in[B \cup\{1\}]$. By virtue of Lemma 3.1 and the fact that $\left[\mathrm{S}_{02} \cup\{0,1\}\right]=\left[\mathrm{S}_{12} \cup\{0,1\}\right]=\left[\mathrm{D}_{1} \cup\{0,1\}\right]=\mathrm{BF}$, it suffices to consider the case $[B]=$ BF. In $[\mathrm{EG} 95]$ it has been shown that the propositional abduction problem remains $\Sigma_{2}^{\mathrm{p}}$-complete when the knowledge base $\Gamma$ is a set of clauses. From such an instance $(\Gamma, A, q)$ we build an instance of $\operatorname{ABD}(B, \mathrm{PQ})$ by rewriting first each clause as an $\vee$-tree of logarithmic depth and then replacing the occurring connectives $\vee$ and $\neg$ by their $B$-representation, thus concluding the proof.

\subsection{Variants of $\operatorname{Abd}(B)$}

We now consider the symmetric abduction problem for different variants on the manifestations: clause, term and $B$-formula. Let us first make a remark on the cases where the manifestation is a (not necessarily positive) literal or a negative literal. 
Remark 4.7. $\operatorname{ABD}(B, Q)$ obeys the same classification as $\operatorname{ABD}(B, P Q)$ since all bounds, upper and lower, easily carry over. $\operatorname{For} \operatorname{ABD}(B, N Q)$ the problem becomes trivial if $[B] \subseteq \mathrm{M}$. For $[B] \subseteq \mathrm{L} \operatorname{ABD}(B, \mathrm{NQ})$ is solvable in polynomial time according to [Zan03]. For the remaining clones (i.e., for $\mathrm{S}_{02} \subseteq[B], \mathrm{S}_{12} \subseteq[B]$, and $\left.\mathrm{D}_{1} \subseteq[B]\right)$, we can again easily adapt the proofs of $\operatorname{ABD}(B, \mathrm{PQ})$. This way we obtain a dichotomous classification for $\operatorname{ABD}(B, N Q)$ into P-complete and $\Sigma_{2}^{\mathrm{p}}$-complete cases; thus skipping the intermediate NP level.

For clauses, it is obvious that $\operatorname{ABD}(B, P Q) \leq_{\mathrm{m}}^{\log } \operatorname{ABD}(B, \mathrm{PC})$. Therefore, all hardness results continue to hold for the $\operatorname{ABD}(B, \mathrm{PC})$. It is an easy exercise to prove that all algorithms that have been developed for a single query can be naturally extended to clauses. Therefore, the complexity classifications for the problems $\operatorname{ABD}(B, \mathrm{PC}), \operatorname{ABD}(B, C)$ and $\operatorname{ABD}(B, N C)$ are exactly the same as for $\operatorname{ABD}(B, \mathrm{PQ}), \operatorname{ABD}(B, \mathrm{Q})$ and $\operatorname{ABD}(B, \mathrm{NQ})$, respectively.

Theorem 4.8. Let $B$ be a finite set of Boolean functions. Then the symmetric abduction problem for propositional B-formulae with a positive clause manifestation, $\operatorname{ABD}(B, \mathrm{PC})$, is

1. $\Sigma_{2}^{\mathrm{p}}$-complete if $\mathrm{S}_{02} \subseteq[B]$ or $\mathrm{S}_{12} \subseteq[B]$ or $\mathrm{D}_{1} \subseteq[B]$,

2. $\mathrm{NP}$-complete if $\mathrm{S}_{00} \subseteq[B] \subseteq \mathrm{M}$ or $\mathrm{S}_{10} \subseteq[B] \subseteq \mathrm{M}$ or $\mathrm{D}_{2} \subseteq[B] \subseteq \mathrm{M}$,

3. in $\mathrm{P}$ and $\oplus$ LOGSPACE-hard if $\mathrm{L}_{2} \subseteq[B] \subseteq \mathrm{L}$, and

4. in LOGSPACE in all other cases.

Notably, we will prove in the next section that allowing for terms as manifestations increases the complexity for the clones $\mathrm{V}$ (from membership in LOGSPACE to NP-completeness), while allowing $B$-formulae as manifestations makes the classification dichotomous again: all problems become either $\mathrm{P}$ - or $\Sigma_{2}^{\mathrm{p}}$-complete.

\section{The complexity of $\operatorname{Abd}(B, P T)$}

Proposition 4.9. Let $B$ be a finite set of Boolean functions such that $\mathrm{V}_{2} \subseteq$ $[B] \subseteq \mathrm{V}$. Then $\operatorname{ABD}(B, \mathrm{PT})$ is $\mathrm{NP}$-complete.

Proof. Let $B$ be a finite set of Boolean functions such that $\mathrm{V}_{2} \subseteq[B] \subseteq \mathrm{V}$ and let $\mathcal{P}=(\Gamma, A, t)$ be an instance of $\operatorname{ABD}(B, \mathrm{PT})$. Hence, $\Gamma$ is a set of $B$-formulae and $t$ is a term, $t=\bigwedge_{i=1}^{n} l_{i}$. Observe that $E$ is a solution for $\mathcal{P}$ if $\Gamma \wedge E$ is satisfiable and for every $i=1, \ldots, n, \Gamma \wedge E \wedge \neg l_{i}$ is not. Given a set $E \subseteq \operatorname{Lits}(A)$, these verifications, which require substitution of symbols and evaluation of an $\checkmark$-formula, can be performed in polynomial time, thus proving membership in NP.

To prove NP-hardness, we give a reduction from 3SAT. Let $\varphi$ be a 3-CNFformula, $\varphi:=\bigwedge_{i \in I} c_{i}$. Let $x_{1}, \ldots, x_{n}$ enumerate the variables occurring in $\varphi$. Let $x_{1}^{\prime}, \ldots, x_{n}^{\prime}$ and $q_{1}, \ldots, q_{n}$ be fresh, pairwise distinct variables. We map $\varphi$ to 
$\mathcal{P}=(\Gamma, A, t)$, where

$$
\begin{aligned}
\Gamma & :=\left\{c_{i}\left[\neg x_{1} / x_{1}^{\prime}, \ldots, \neg x_{n} / x_{n}^{\prime}\right] \mid i \in I\right\} \\
& \cup\left\{x_{i} \vee x_{i}^{\prime}, x_{i} \vee q_{i}, x_{i}^{\prime} \vee q_{i} \mid 1 \leq i \leq n\right\}, \\
A & :=\left\{x_{1}, \ldots, x_{n}, x_{1}^{\prime}, \ldots, x_{n}^{\prime}\right\}, \\
t & :=q_{1} \wedge \cdots \wedge q_{n} .
\end{aligned}
$$

We show that $\varphi$ is satisfiable if and only if $\mathcal{P}$ has a solution. First assume that $\varphi$ is satisfied by the assignment $\sigma:\left\{x_{1}, \ldots, x_{n}\right\} \longrightarrow\{0,1\}$. Define $E:=\left\{\neg x_{i} \mid\right.$ $\left.\sigma\left(x_{i}\right)=0\right\} \cup\left\{\neg x_{i}^{\prime} \mid \sigma\left(x_{i}\right)=1\right\}$ and $\hat{\sigma}$ as the extension of $\sigma$ mapping $\hat{\sigma}\left(x_{i}^{\prime}\right)=$ $\neg \sigma\left(x_{i}\right)$ and $\hat{\sigma}\left(q_{i}\right)=1$ for all $1 \leq i \leq n$. Obviously, $\hat{\sigma} \models \Gamma \wedge E$. Furthermore, $\Gamma \wedge E \models q_{i}$ for all $1 \leq i \leq n$, because any satisfying assignment of $\Gamma \wedge E$ sets to 0 either $x_{i}$ or $x_{i}^{\prime}$ and thus $\left\{x_{i} \vee q_{i}, x_{i}^{\prime} \vee q_{i}\right\} \models q_{i}$. Hence $E$ is an explanation for $\mathcal{P}$.

Conversely, suppose that $\mathcal{P}$ has a full explanation $E$. The facts that $\Gamma \wedge$ $E \models q_{1} \wedge \cdots \wedge q_{n}$ and that each $q_{i}$ occurs only in the clauses $x_{i} \vee q_{i}, x_{i}^{\prime} \vee q_{i}$ enforce that, for every $i, E$ contains $\neg x_{i}$ or $\neg x_{i}^{\prime}$. Because of the clause $x_{i} \vee x_{i}^{\prime}$, it cannot contain both. Therefore in $E$ the value of $x_{i}^{\prime}$ is determined by the value of $x_{i}$ and is its dual. From this it is easy to conclude that the assignment $\sigma:\left\{x_{1}, \ldots, x_{n}\right\} \longrightarrow\{0,1\}$ defined by $\sigma\left(x_{i}\right)=0$ if $\neg x_{i} \in E$, and 1 otherwise, satisfies $\varphi$. Finally $\mathcal{P}$ can be transformed into an $\operatorname{ABD}(B, \mathrm{PT})$-instance, because every formula in $\Gamma$ is the disjunction of at most three variables and $\vee \in[B]$.

Theorem 4.10. Let $B$ be a finite set of Boolean functions. Then the symmetric abduction problem for propositional B-formulae with a positive term manifestation, $\operatorname{ABD}(B, \mathrm{PT})$, is

1. $\Sigma_{2}^{\mathrm{p}}$-complete if $\mathrm{S}_{02} \subseteq[B]$ or $\mathrm{S}_{12} \subseteq[B]$ or $\mathrm{D}_{1} \subseteq[B]$,

2. NP-complete if $\mathrm{V}_{2} \subseteq[B] \subseteq \mathrm{M}$ or $\mathrm{S}_{10} \subseteq[B] \subseteq \mathrm{M}$ or $\mathrm{D}_{2} \subseteq[B] \subseteq \mathrm{M}$,

3. in $\mathrm{P}$ and $\oplus$ LOGSPACE-hard if $\mathrm{L}_{2} \subseteq[B] \subseteq \mathrm{L}$, and

4. in LOGSPACE in all other cases.

Proof. 1. The $\Sigma_{2}^{\mathrm{p}}$-hardness follows directly from Proposition 4.6.

2. For the clones $\mathrm{V}_{2} \subseteq[B] \subseteq \mathrm{V}$, see Proposition 4.9. In all other clones, the NP-hardness follows from a straightforward generalization of the proof of Proposition 4.5.

3. Membership in P follows directly from [NZ08, Theorem 67], the $\oplus$ LOGSPACEhardness from Proposition 4.4.

4. Analogous to Proposition 4.3.

Remark 4.11. All upper and lower bounds for $\operatorname{ABD}(B, P T)$ easily carry over to $\operatorname{ABD}(B, \mathrm{~T})$. It is also easily seen that $\operatorname{ABD}(B, \mathrm{NT})$ is classified exactly as $\operatorname{ABd}(B, N Q)$, see Remark 4.7.

The complexity of $\operatorname{Abd}(B, \mathcal{L}(B))$ 
Proposition 4.12. Let $B$ be a finite set of Boolean functions such that $\mathrm{S}_{00} \subseteq$ $[B]$ or $\mathrm{S}_{10} \subseteq[B]$ or $\mathrm{D}_{2} \subseteq[B]$. Then $\operatorname{ABD}(B, \mathcal{L}(B))$ is $\Sigma_{2}^{\mathrm{p}}$-complete.

Proof. We prove $\Sigma_{2}^{\mathrm{p}}$-hardness by giving a reduction from the $\Sigma_{2}^{\mathrm{p}}$-hard problem $\operatorname{QSAT}_{2}$ [Wra77]. Let an instance of $\mathrm{QSAT}_{2}$ be given by a closed formula $\chi:=\exists x_{1} \cdots \exists x_{n} \forall y_{1} \cdots \forall y_{m} \varphi$ with $\varphi$ being a 3 -DNF-formula. First observe that $\exists x_{1} \cdots \exists x_{n} \forall y_{1} \cdots \forall y_{m} \varphi$ is true if and only if there exists a consistent set $X \subseteq$ $\operatorname{Lits}\left(\left\{x_{1}, \ldots, x_{n}\right\}\right)$ such that $X \cap\left\{x_{i}, \neg x_{i}\right\} \neq \emptyset$, for all $1 \leq i \leq n$, and $\neg X \vee \varphi$ is (universally) valid (or equivalently $\neg \varphi \wedge X$ is unsatisfiable).

Denote by $\bar{\varphi}$ the negation normal form of $\neg \varphi$ and let $\bar{\varphi}^{\prime}$ be obtained from $\bar{\varphi}$ by replacing all occurrences of $\neg x_{i}$ with a fresh proposition $x_{i}^{\prime}, 1 \leq i \leq n$, and all occurrences of $\neg y_{i}$ with a fresh proposition $y_{i}^{\prime}, 1 \leq i \leq m$. That is, $\bar{\varphi}^{\prime} \equiv \bar{\varphi}\left[\neg x_{1} / x_{1}^{\prime}, \ldots, \neg x_{n} / x_{n}^{\prime}, \neg y_{1} / y_{1}^{\prime}, \ldots, \neg y_{m} / y_{m}^{\prime}\right]$. Thus $\bar{\varphi}^{\prime}=\bigwedge_{i \in I} c_{i}^{\prime}$, where every $c_{i}^{\prime}$ is a disjunction of three propositions. To $\chi$ we associate the propositional abduction problem $\mathcal{P}=(\Gamma, A, \psi)$ defined as follows:

$$
\begin{aligned}
\Gamma: & :=\left\{c_{i}^{\prime} \vee q \mid i \in I\right\} \\
& \cup\left\{x_{i} \vee x_{i}^{\prime} \mid 1 \leq i \leq n\right\} \cup\left\{y_{i} \vee y_{i}^{\prime} \mid 1 \leq i \leq m\right\} \\
& \cup\left\{f_{i} \vee x_{i}, t_{i} \vee x_{i}^{\prime}, f_{i} \vee t_{i} \mid 1 \leq i \leq n\right\}, \\
A: & :=\left\{t_{i}, f_{i} \mid 1 \leq i \leq n\right\}, \\
\psi & :=q \vee \bigvee_{1 \leq i \leq n}\left(x_{i} \wedge x_{i}^{\prime}\right) \vee \bigvee_{1 \leq i \leq m}\left(y_{i} \wedge y_{i}^{\prime}\right) .
\end{aligned}
$$

Suppose that $\chi$ is true. Then there exists an assignment $\sigma:\left\{x_{1}, \ldots, x_{n}\right\} \longrightarrow$ $\{0,1\}$ such that no extension $\sigma^{\prime}:\left\{x_{1}, \ldots, x_{n}\right\} \cup\left\{y_{1}, \ldots, y_{m}\right\} \longrightarrow\{0,1\}$ of $\sigma$ satisfies $\neg \varphi$. Define $X$ as the set of literals over $\left\{x_{1}, \ldots, x_{n}\right\}$ set to 1 by $\sigma$. Defining $E:=\left\{\neg f_{i}, t_{i} \mid x_{i} \in X\right\} \cup\left\{\neg t_{i}, f_{i} \mid \neg x_{i} \in X\right\}$, we obtain with abuse of notation

$$
\begin{aligned}
\Gamma \wedge E \wedge \neg \psi \equiv & \bigwedge_{i \in I} c_{i}^{\prime} \wedge \bigwedge_{1 \leq i \leq n}\left(x_{i} \oplus x_{i}^{\prime}\right) \wedge \bigwedge_{1 \leq i \leq m}\left(y_{i} \oplus y_{i}^{\prime}\right) \wedge \\
& \bigwedge_{1 \leq i \leq n, \sigma\left(x_{i}\right)=1} x_{i} \wedge \bigwedge_{1 \leq i \leq n, \sigma\left(x_{i}\right)=0} x_{i}^{\prime} \\
\equiv & \neg \varphi \wedge X,
\end{aligned}
$$

which is unsatisfiable by assumption. As $\Gamma \wedge E$ is satisfied by any assignment setting in addition all $x_{i}, x_{i}^{\prime}, 1 \leq i \leq n$, and all $y_{j}, y_{j}^{\prime}, 1 \leq i \leq m$, to 1 , we have proved that $E$ is an explanation for $\mathcal{P}$.

Conversely, suppose that $\mathcal{P}$ has an explanation $E$. Assume w.l.o.g. that $E$ is full. Due to the clause $\left(f_{i} \vee t_{i}\right)$ in $\Gamma$, we also may assume that $\left|E \cap\left\{\neg t_{i}, \neg f_{i}\right\}\right| \leq 1$ for all $1 \leq i \leq n$.

Setting $X:=\left\{x_{i} \mid \neg f_{i} \in E\right\} \cup\left\{\neg x_{i} \mid \neg t_{i} \in E\right\}$ we now obtain $\bigwedge_{1 \leq i \leq n}\left(\left(f_{i} \vee\right.\right.$ $\left.\left.x_{i}\right) \wedge\left(t_{i} \vee \neg x_{i}\right) \wedge\left(f_{i} \vee t_{i}\right)\right) \wedge E \equiv X$ and $\Gamma \wedge E \wedge \neg \psi \equiv \neg \varphi \wedge X$ as above. Hence, $\neg \varphi \wedge X$ is unsatisfiable, which implies the existence of an assignment $\sigma:\left\{x_{1}, \ldots, x_{n}\right\} \longrightarrow$ $\{0,1\}$ such that no extension $\sigma^{\prime}:\left\{x_{1}, \ldots, x_{n}\right\} \cup\left\{y_{1}, \ldots, y_{m}\right\} \longrightarrow\{0,1\}$ of $\sigma$ satisfies $\neg \varphi$. Therefore, we have proved that $\chi$ is true if and only if $\mathcal{P}$ has an explanation.

It remains to show that $\mathcal{P}$ can be transformed into an $\operatorname{ABD}(B, \mathcal{L}(B))$-instance for any relevant $B$. Since $\left[\mathrm{S}_{00} \cup\{1\}\right]=\mathrm{S}_{01},\left[\mathrm{~S}_{10} \cup\{1\}\right]=\mathrm{M}_{1},\left[\mathrm{D}_{2} \cup\{1\}\right]=\mathrm{S}_{01}^{2}$ and 
$\mathrm{S}_{01} \subseteq \mathrm{S}_{01}^{2} \subseteq \mathrm{M}_{1}$, it suffices to consider the case $[B]=\mathrm{S}_{01}$ by Lemma 3.1. Observe that $x \vee(y \wedge z) \in[B]$. The transformation can hence be done in polynomial time by local replacements, rewriting $\psi$ as $\bigvee_{1 \leq i \leq n} q \vee\left(x_{i} \wedge x_{i}^{\prime}\right) \vee \bigvee_{1 \leq i \leq m} q \vee\left(y_{i} \wedge y_{i}^{\prime}\right)$ and using the associativity of $\mathrm{V}$.

Theorem 4.13. Let $B$ be a finite set of Boolean functions. Then the symmetric abduction problem for propositional B-formulae with a B-formula manifestation, $\operatorname{ABD}(B, \mathcal{L}(B))$, is

1. $\Sigma_{2}^{\mathrm{p}}$-complete if $\mathrm{S}_{00} \subseteq[B]$ or $\mathrm{S}_{10} \subseteq[B]$ or $\mathrm{D}_{2} \subseteq[B]$,

2. in $\mathrm{P}$ and $\oplus$ LOGSPACE-hard if $\mathrm{L}_{2} \subseteq[B] \subseteq \mathrm{L}$, and

3. in LOGSPACE in all other cases.

Proof. 1. See Proposition 4.12.

2. Membership in $\mathrm{P}$ follows directly from [Zan03], the $\oplus$ LOGSPACE-hardness follows from Proposition 4.4.

3. Analogous to Proposition 4.3.

Observe that there are no sets $B$ of Boolean functions for which $\operatorname{ABD}(B, \mathcal{L}(B))$ is NP-complete.

\section{The complexity of P-Abd(B)}

We will now study the complexity of positive abduction, in which an explanation consists of a set of positive literals. The results of this section are summarized in Figure 2. To begin with, note that for monotonic or 1-reproducing sets of formulae, deciding the existence of a positive explanation reduces to a testing whether $A$ is one.

Lemma 5.1. For $[B] \subseteq \mathrm{R}_{1}$ or $[B] \subseteq \mathrm{M}$, an instance $(\Gamma, A, \varphi)$ of $\mathrm{P}-\mathrm{ABD}(B, \mathcal{M})$ has solutions if and only if $A$ is a solution.

Proof. Let $E \subseteq A$ be an arbitrary explanation for the given instance $(\Gamma, A, \varphi)$. One easily verifies that $\Gamma \wedge A$ remains satisfiable if $\Gamma \wedge E$ was, and $\Gamma \wedge A \wedge \neg \varphi$ remains unsatisfiable if $\Gamma \wedge E \wedge \neg \varphi$ was. Conversely, if $A$ is not an explanation, no proper subset $E \subseteq A$ can be an explanation either.

As a consequence we will see that some of the formerly NP-complete cases become tractable and that some of the formerly $\Sigma_{2}^{\mathrm{p}}$-complete cases become coNPcomplete.

\subsection{The complexity of $\mathrm{P}-\mathrm{Abd}(B, \mathrm{PQ})$}

Proposition 5.2. Let $[B] \subseteq \mathrm{M}$. Then $\mathrm{P}-\mathrm{AbD}(B, \mathrm{PQ}) \in$ Logspace.

Proof. According to Lemma 5.1 it suffices to test if $A$ is a solution, that is, to test if $\Gamma \wedge A \neg q$ or equivalently $\Gamma \wedge A[q / 0]$ is unsatisfiable. This can be done in logarithmic space, since $\Gamma \wedge A$ is a monotonic formula [Lew79]. 
Proposition 5.3. Let $\mathrm{S}_{02} \subseteq[B] \subseteq \mathrm{R}_{1}$ or $\mathrm{S}_{12} \subseteq[B] \subseteq \mathrm{R}_{1}$ or $\mathrm{D}_{1} \subseteq[B] \subseteq \mathrm{R}_{1}$. Then $\mathrm{P}-\mathrm{ABD}(B, \mathrm{PQ})$ is coNP-complete.

Proof. According to Lemma 5.1 it suffices to test whether $A$ is a solution. Since $\Gamma \wedge A$ is always satisfiable, only the task of testing whether $\Gamma \wedge A \wedge \neg q$ is unsatisfiable remains. And this can be done in coNP.

Since $\left[\mathrm{D}_{1} \cup\{1\}\right]=\left[\mathrm{S}_{12} \cup\{1\}\right]=\mathrm{R}_{1},\left[\mathrm{~S}_{02} \cup\{1\}\right]=\mathrm{S}_{0}$ and $\mathrm{S}_{0} \subseteq \mathrm{R}_{1}$, it suffices to show hardness for the case $\mathrm{S}_{0} \subseteq[B]$ by Lemma 3.1. To show coNPhardness we give a reduction from $\overline{3 \mathrm{SAT}}$. Let $\varphi=\bigwedge_{i \in I} c_{i}$ be a 3-CNF-formula, i.e., each clause $c_{i}$ consists of the disjunction of exactly three literals. Since $[\{\rightarrow, 0\}]=\mathrm{BF}$, each clause $c_{i}$ has a representation as a $\{\rightarrow, 0\}$-formula which we indicate by $c_{i}^{\prime}$. Let $q$ be a fresh proposition. We map $\varphi$ to $(\Gamma, \emptyset, q)$, where we define $\Gamma=\bigcup_{i \in I} c_{i}^{\prime}[0 / q]$. Note that $\Gamma$ is a set of $\mathrm{S}_{0}$-formulae of polynomial size and 1reproducing. Let $\varphi$ be unsatisfiable. Then $\Gamma$ is satisfied by the assignment setting to 1 all propositions and $\Gamma \wedge \neg q$ is unsatisfiable, because it is equivalent to $\varphi \wedge \neg q$. Summing up, $\emptyset$ is an explanation for $(\Gamma, \emptyset, q)$. Conversely, let $\varphi$ be satisfiable. This implies that $\Gamma \wedge \neg q$ is satisfiable and thus $(\Gamma, \emptyset, q)$ has no explanations.

It remains to transform $(\Gamma, \emptyset, q)$ into a $\mathrm{P}-\mathrm{ABD}(B, \mathrm{PQ})$-instance for any relevant $B$. As $\rightarrow \in \mathrm{S}_{0} \subseteq[B]$, this is done by replacing in $\Gamma$ every occurrence of $\rightarrow$ by its $B$-representation.

Theorem 5.4. Let $B$ be a finite set of Boolean functions. Then the positive abduction problem for propositional B-formulae with a positive literal manifestation, $\mathrm{P}-\mathrm{ABD}(B, \mathrm{PQ})$, is

1. $\Sigma_{2}^{\mathrm{p}}$-complete if $\mathrm{D} \subseteq[B]$ or $\mathrm{S}_{1} \subseteq[B]$,

2. coNP-complete if $\mathrm{S}_{02} \subseteq[B] \subseteq \mathrm{R}_{1}$ or $\mathrm{S}_{12} \subseteq[B] \subseteq \mathrm{R}_{1}$ or $\mathrm{D}_{1} \subseteq[B] \subseteq \mathrm{R}_{1}$,

3. in $\mathrm{P}$ and $\oplus$ LOGSPACE-hard if $\mathrm{L}_{2} \subseteq[B] \subseteq \mathrm{L}$,

4. in LOGSPACE in all other cases.

Proof. 1. In [NZ08], Nordh and Zanuttini prove that the abduction problem in which the knowledge base is a set of clauses remains $\Sigma_{2}^{\mathrm{p}}$-hard even if explanations are required to comprise positive literals only. A reduction from this problem can be done analogously to the one in Proposition 4.6.

2. See Proposition 5.3.

3. Membership in $\mathrm{P}$ follows from [NZ08, Theorem 66]. For the $\oplus$ LOGSPACEhardness the same reduction as in Proposition 4.4 works.

4. See Proposition 5.2.

\subsection{Variants of $\mathrm{P}-\mathrm{Abd}(B)$}

Having examined the complexity of positive abduction for positive literal manifestations, we will now consider positive abduction for manifestation that are restricted to be respectively a clause, a term, or a $B$-formula. But first let us make a remark on the complexity of positive abduction when the manifestation is a (not necessarily positive) literal or a negative literal. 
Remark 5.5. Again all upper and lower bounds for $\mathrm{P}-\mathrm{ABD}(B, \mathrm{PQ})$ easily carry over to $\mathrm{P}-\mathrm{ABD}(B, \mathrm{Q})$. For $\mathrm{P}-\mathrm{ABD}(B, \mathrm{NQ})$ the problem becomes trivial if $[B] \subseteq \mathrm{R}_{1}$ (Lemma 5.1). For $\mathrm{L}_{0} \subseteq[B] \subseteq \mathrm{L}$ and $\mathrm{L}_{3} \subseteq[B] \subseteq \mathrm{L}$, we obtain membership in $\mathrm{P}$ from [NZ08, Theorem 66]. For all remaining cases (i.e., for $\mathrm{D} \subseteq[B]$ and $\mathrm{S}_{1} \subseteq[B]$ ), we obtain $\Sigma_{2}^{\mathrm{p}}$-completeness from an easy adaption of the first part in the proof of Proposition 5.4.

Analogously to the symmetric case the algorithms can be extended to clauses. Thus, $\mathrm{P}-\mathrm{ABD}(B, \mathrm{PC})$ is classified as $\mathrm{P}-\mathrm{ABD}(B, \mathrm{PQ})$. Similarly the classifications for $\mathrm{P}-\mathrm{ABD}(B, \mathrm{C})$ and $\mathrm{P}-\mathrm{ABD}(B, \mathrm{NC})$ are the same as classifications for $\mathrm{P}-\operatorname{ABD}(B, \mathrm{Q})$ and $\mathrm{P}-\mathrm{ABD}(B, \mathrm{NQ})$.

Theorem 5.6. Let $B$ be a finite set of Boolean functions. Then the positive abduction problem for propositional $B$-formulae with a positive clause manifestation, $\mathrm{P}-\mathrm{ABD}(B, \mathrm{PC})$, is

1. $\Sigma_{2}^{\mathrm{p}}$-complete if $\mathrm{D} \subseteq[B]$ or $\mathrm{S}_{1} \subseteq[B]$,

2. coNP-complete if $\overline{\mathrm{S}}_{02} \subseteq[B] \subseteq \mathrm{R}_{1}$ or $\mathrm{S}_{12} \subseteq[B] \subseteq \mathrm{R}_{1}$ or $\mathrm{D}_{1} \subseteq[B] \subseteq \mathrm{R}_{1}$,

3. in $\mathrm{P}$ and $\oplus$ LOGSPACE-hard if $\mathrm{L}_{2} \subseteq[B] \subseteq \mathrm{L}$,

4. in LOGSPACE in all other cases.

The Complexity of $\mathbf{P}-\mathbf{A b d}(\boldsymbol{B}, \mathbf{P T})$ The classification for positive terms is identical to the one for a single positive literal, except for the affine clones $L_{0}$, $\mathrm{L}_{3}$, and $\mathrm{L}$. For these, the complexity of $\mathrm{P}-\mathrm{ABD}(B, \mathrm{PT})$ jump from membership in $\mathrm{P}$ to NP-completeness.

Proposition 5.7. Let $\mathrm{L}_{0} \subseteq[B] \subseteq \mathrm{L}$ or $\mathrm{L}_{3} \subseteq[B] \subseteq \mathrm{L}$. Then $\mathrm{P}-\mathrm{ABD}(B, \mathrm{PT})$ is NP-complete.

Proof. Let $(\Gamma, A, t)$ with $t=\bigwedge_{i \in I} x_{i}$ be an instance of $\mathrm{P}-\mathrm{ABD}(B, \mathrm{PT})$. To check whether a given $E \subseteq A$ is an explanation, we have to test the satisfiability of $\Gamma \wedge E$ and the unsatisfiability of $\Gamma \wedge E \wedge \neg x_{i}$ for every $i \in I$. These tasks are equivalent to solving systems of linear equations, which can be done in polynomial time. The hardness follows directly from [NZ08, Theorem 70].

Theorem 5.8. Let $B$ be a finite set of Boolean functions. Then the positive abduction problem for propositional B-formulae with a positive term manifestation, $\mathrm{P}-\mathrm{ABD}(B, \mathrm{PT})$, is

1. $\Sigma_{2}^{\mathrm{p}}$-complete if $\mathrm{D} \subseteq[B]$ or $\mathrm{S}_{1} \subseteq[B]$,

2. coNP-complete if $\mathrm{S}_{02} \subseteq[B] \subseteq \mathrm{R}_{1}$ or $\mathrm{S}_{12} \subseteq[B] \subseteq \mathrm{R}_{1}$ or $\mathrm{D}_{1} \subseteq[B] \subseteq \mathrm{R}_{1}$,

3. NP-complete if $[B] \in\left\{\mathrm{L}, \mathrm{L}_{0}, \mathrm{~L}_{3}\right\}$,

4. in $\mathrm{P}$ and $\oplus$ LOGSPACE-hard if $[B] \in\left\{\mathrm{L}_{1}, \mathrm{~L}_{2}\right\}$,

5. in LOGSPACE in all other cases.

Proof. 1. Follows from the first item of Proposition 5.4.

2. Both membership and hardness follow from Proposition 5.3.

3. See Proposition 5.7. 
4. For the $\oplus$ LOGSPACE-hardness the same reduction as in Proposition 4.4 works. Since $\mathrm{L}_{1} \subseteq \mathrm{R}_{1}$, according to Lemma 5.1, it suffices to check whether $A$ is a solution. This task reduces to solve systems of linear equations which is in P.

5. Analogously to Proposition 5.2.

Remark 5.9. Again all upper and lower bounds for $\mathrm{P}-\mathrm{ABD}(B, \mathrm{PT})$ carry over to $\mathrm{P}-\mathrm{ABD}(B, \mathrm{~T})$. For $\mathrm{P}-\mathrm{ABD}(B, \mathrm{NT})$ the problem becomes trivial if $[B] \subseteq \mathrm{R}_{1}$ (Lemma 5.1). For $[B] \in\left\{\mathrm{L}, \mathrm{L}_{0}, \mathrm{~L}_{3}\right\}$, we obtain NP-completeness with the hardness being obtained from an easy reduction from $\mathrm{P}-\operatorname{ABD}(B, \mathrm{PT})$ : as we have $x \oplus y \in$ $[B \cup\{1\}]$, we can simply transform the given positive term into a negative one. For the remaining cases (i.e., $\mathrm{D} \subseteq[B]$ and $\mathrm{S}_{1} \subseteq[B]$ ), we obtain $\Sigma_{2}^{\mathrm{p}}$-completeness from an adaption of the first part in the proof of Proposition 5.4.

The Complexity of P-Abd $(\boldsymbol{B}, \mathcal{L}(\boldsymbol{B}))$ The complexity of P-ABD $(B, \mathcal{L}(B))$ differs from the complexity of $\mathrm{P}-\mathrm{ABD}(B, \mathrm{PQ})$ for the clones either (a) above $\mathrm{E}$ or $V$ and below $M$ or (b) above $L_{0}$ or $L_{3}$ and below $L$. For the former the complexity increases to coNP-completeness, whereas for the latter we obtain membership in $\mathrm{NP}$ and hardness for $\oplus$ LOGSPACE; the exact complexity of positive abduction when both the knowledge base and the manifestation are represented by non-1reproducing affine formulae remains an open problem.

Proposition 5.10. Let $B$ be a finite set of Boolean functions such that $\mathrm{L}_{0} \subseteq$ $[B] \subseteq \mathrm{L}$ or $\mathrm{L}_{3} \subseteq[B] \subseteq \mathrm{L}$. Then $\mathrm{P}-\mathrm{ABD}(B, \mathcal{L}(B)) \in \mathrm{NP}$.

Proof. Let $E \subseteq A$ be a potential solution. The test for satisfiability of $\Gamma \wedge E$ and the test for unsatisfiability of $\Gamma \wedge E \wedge \neg \varphi$ are equivalent to solving two systems of linear equations, which can be done in polynomial time.

Proposition 5.11. Let $B$ be a finite set of Boolean functions such that $\mathrm{S}_{00} \subseteq$ $[B] \subseteq \mathrm{M}$ or $\mathrm{S}_{10} \subseteq[B] \subseteq \mathrm{M}$ or $\mathrm{D}_{2} \subseteq[B] \subseteq \mathrm{M}$. Then $\mathrm{P}-\mathrm{ABD}(B, \mathcal{L}(B))$ is coNPcomplete.

Proof. We will first prove coNP-membership. According to Lemma 5.1, it suffices to test whether $A$ is a solution. This can be done by first verifying that $\Gamma \wedge A$ is satisfiable, and afterwards verifying that $\Gamma \wedge A \wedge \neg \varphi$ is unsatisfiable. As $\Gamma$ is a set of monotonic formulae, deciding the satisfiability of $\Gamma \wedge A$ can be done in logarithmic space; and deciding whether $\Gamma \wedge A \wedge \neg \varphi$ is unsatisfiable is in coNP.

To establish coNP-hardness, we give a reduction from the coNP-hard problem to decide whether a given 3-DNF-formulae $\varphi$ is a tautology. Let $\operatorname{Vars}(\varphi)=$ $\left\{x_{1}, \ldots x_{n}\right\}$. Denote by $\bar{\varphi}$ the negation normal form of $\neg \varphi$ and let $\bar{\varphi}^{\prime}$ be obtained from $\bar{\varphi}$ by replacing all occurrences of $\neg x_{i}$ with a fresh proposition $x_{i}^{\prime}, 1 \leq i \leq n$. That is, $\bar{\varphi}^{\prime} \equiv \bar{\varphi}\left[\neg x_{1} / x_{1}^{\prime}, \ldots, \neg x_{n} / x_{n}^{\prime}\right]$. Thus $\bar{\varphi}^{\prime}=\bigwedge_{i \in I} c_{i}^{\prime}$ where every $c_{i}^{\prime}$ is a disjunction of three propositions. To $\varphi$ we associate the propositional abduction problem $\mathcal{P}=(\Gamma, \emptyset, \psi)$ defined as follows:

$$
\begin{aligned}
& \Gamma:=\left\{c_{i}^{\prime} \vee q \mid i \in I\right\} \cup\left\{x_{i} \vee x_{i}^{\prime} \mid 1 \leq i \leq n\right\}, \\
& \psi:=q \vee \bigvee_{1 \leq i \leq n}\left(x_{i} \wedge x_{i}^{\prime}\right) .
\end{aligned}
$$


Observe that

$$
\Gamma \wedge \neg \psi \equiv \neg \varphi \wedge \neg q \wedge \bigwedge_{i=1}^{n}\left(x_{i} \oplus x_{i}^{\prime}\right) .
$$

Suppose that $\varphi$ is a tautology, i.e., $\neg \varphi$ is unsatisfiable. From (4) it follows that $\Gamma \wedge \neg \psi$ is unsatisfiable. As $\Gamma$ is satisfiable, $\emptyset$ is a solution for $\mathcal{P}$.

Suppose conversely that $\emptyset$ is a solution for $\mathcal{P}$. Then $\Gamma \wedge \neg \psi \equiv \neg \varphi \wedge \neg q \wedge$ $\bigwedge_{i=1}^{n}\left(x_{i} \oplus x_{i}^{\prime}\right)$ is unsatisfiable. Since $q$ and the $x_{i}^{\prime}$ do not occur in $\varphi$, we obtain the unsatisfiability of $\neg \varphi$. Hence, $\varphi$ is a tautology.

The transformation of $\mathcal{P}$ into an $\mathrm{P}-\operatorname{ABD}(B, \mathcal{L}(B))$-instance for any relevant $B$ can be done in exactly the same way as in the proof of Proposition 4.12.

Theorem 5.12. Let $B$ be a finite set of Boolean functions. Then the positive abduction problem for propositional B-formulae with a B-formula manifestation, $\mathrm{P}-\operatorname{ABD}(B, \mathcal{L}(B))$, is

1. $\Sigma_{2}^{\mathrm{p}}$-complete if $\mathrm{D} \subseteq[B]$ or $\mathrm{S}_{1} \subseteq[B]$,

2. coNP-complete if $\mathrm{S}_{02} \subseteq[B] \subseteq \mathrm{R}_{1}$ or $\mathrm{S}_{12} \subseteq[B] \subseteq \mathrm{R}_{1}$ or $\mathrm{D}_{1} \subseteq[B] \subseteq \mathrm{R}_{1}$ or $\mathrm{S}_{00} \subseteq[B] \subseteq \mathrm{M}$ or $\mathrm{S}_{10} \subseteq[B] \subseteq \mathrm{M}$ or $\mathrm{D}_{2} \subseteq[B] \subseteq \mathrm{M}$

3. in $\mathrm{NP}$ and $\oplus$ LOGSPACE-hard if $[B] \in\left\{\mathrm{L}, \mathrm{L}_{0}, \mathrm{~L}_{3}\right\}$,

4. in $\mathrm{P}$ and $\oplus$ LOGSPACE-hard if $[B] \in\left\{\mathrm{L}_{1}, \mathrm{~L}_{2}\right\}$,

5. in LOGSPACE in all other cases.

Proof. 1. Follows from the first item of Proposition 5.4.

2. See Proposition 5.11 and 5.3.

3. For membership in NP, see Proposition 5.10. The $\oplus$ LogsPaCE-hardness, on the other hand, is established using the same reduction as in the proof of Proposition 4.4.

4. For membership in $\mathrm{P}$, see the fourth item of Proposition 5.8. The $\oplus$ LOGSPACEhardness follows from Proposition 4.4 as above.

5. For $[B] \subseteq \mathrm{V}$, a $B$-formula is a positive clause. Thus the result follows from Theorem 5.6. For $[B] \subseteq \mathrm{N}$ and $[B] \subseteq \mathrm{E}$, see Proposition 4.3.

\section{Overview of Results}

The following two tables give an overview of the results for the studied symmetric and positive abduction problems. The small numbers on the right side in the table cells refer to the corresponding theorem/proposition/remark. The number is omitted for trivial results. 


\begin{tabular}{|c|c|c|c|c|c|c|c|}
\hline Manifestation & $\mathrm{E}_{*}$ & $\mathrm{~N}_{*}$ & $\mathrm{~V}_{*}$ & & $\mathrm{~L}_{*}$ & $\begin{array}{c}\mathrm{D}_{2}, \mathrm{~S}_{* 0} \subseteq \\
{[B] \subseteq \mathrm{M}}\end{array}$ & $\begin{array}{l}\mathrm{D}_{1}, \mathrm{~S}_{* 2} \subseteq \\
{[B] \subseteq \mathrm{BF}}\end{array}$ \\
\hline $\mathrm{NQ}, \mathrm{NC}, \mathrm{NT}$ & $\in \mathrm{L} \quad 4.7$ & $\in \mathrm{L} \quad 4.7$ & $\in \mathrm{L}$ & 4.7 & $\in \mathrm{P} \quad 4.4$ & $\in \mathrm{L} \quad 4.7$ & $\Sigma_{2}^{\mathrm{p}}$-c. $\quad 4.7$ \\
\hline $\mathrm{PQ}, \mathrm{PC}, \mathrm{Q}, \mathrm{C}$ & $\in \mathrm{L} \quad 4.3$ & $\in \mathrm{L} \quad 4.3$ & $\in \mathrm{L}$ & 4.3 & $\in \mathrm{P} \quad 4.4$ & NP-c. 4.5 & $\Sigma_{2}^{\mathrm{p}}$-c. $\quad 4.6$ \\
\hline $\mathrm{PT}, \mathrm{T}$ & $\in \mathrm{L} \quad 4.10$ & $\in \mathrm{L} \quad 4.10$ & NP-c. & 4.9 & $\in \mathrm{P} \quad 4.10$ & NP-c. 4.10 & $\Sigma_{2}^{\mathrm{p}}$-c. $\quad 4.10$ \\
\hline $\mathcal{L}(B)$ & $\in \mathrm{L} \quad 4.13$ & $\in \mathrm{L} \quad 4.13$ & $\in \mathrm{L}$ & 4.13 & $\in \mathrm{P} \quad 4.13$ & $\Sigma_{2}^{\mathrm{p}}$-c. $\quad 4.12$ & $\Sigma_{2}^{\mathrm{p}}$-c. 4.12 \\
\hline
\end{tabular}

Table 2. The complexity of ABD, where $*$-subscripts on clones denote all valid completions, L abbreviates LOGSPACE, and the suffix "-c." indicates completeness for the respective complexity class.

\begin{tabular}{|c|c|c|c|c|c|c|}
\hline $\operatorname{Ian}$ & $\begin{array}{c}\mathrm{E}_{*}, \mathrm{~N}_{*}, \\
\mathrm{~V}_{*},\end{array}$ & $\mathrm{~L}_{1}, \mathrm{~L}_{2}$ & $\mathrm{~L}_{0}, \mathrm{~L}_{3}, \mathrm{~L}$ & $\begin{array}{c}\mathrm{D}_{2}, \mathrm{~S}_{* 0} \subseteq \\
{[B] \subseteq \mathrm{M}}\end{array}$ & $\begin{array}{l}\mathrm{D}_{1}, \mathrm{~S}_{* 2} \subseteq \\
{[B] \subseteq \mathrm{R}_{1}}\end{array}$ & $\begin{array}{c}\mathrm{D}, \mathrm{S}_{1} \subseteq \\
{[B] \subseteq \mathrm{BF}}\end{array}$ \\
\hline$\psi$ & $\in \mathrm{L}$ & $\in \mathrm{L}$ & $\in \mathrm{P} \quad 5.5$ & $\in \mathrm{L}$ & $\in \mathrm{L}$ & $\sum_{2}^{\mathrm{p}}$-c. 5 \\
\hline $\mathrm{T}$ & L & $\in \mathrm{L}$ & NP-c. 5.9 & $\in \mathrm{L}$ & $\epsilon$ & $\Sigma_{2}^{\mathrm{p}}$-c. 5.9 \\
\hline Q, & $\in$ & $\in \mathrm{P}_{5}$ & $\in \mathrm{P} \quad 5.4$ & $\in \mathrm{L}$ & $\mathrm{coN}$ & $\Sigma_{2}^{\mathrm{p}}$-c. \\
\hline 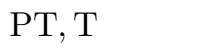 & $\epsilon$ & $\in \mathrm{P} \quad 5.8$ & NP-c. 5.7 & $\in \mathrm{L}$ & coNP-c. 5.8 & $\Sigma_{2}^{\mathrm{p}}$-c. \\
\hline$(B)$ & $\in \mathrm{L}$ & $\in \mathrm{P}_{5.12}$ & $\in \mathrm{NP} 5.10$ & coNP-c. 5. & coNP-c. 5.12 & $\Sigma_{2}^{\mathrm{p}} \mathrm{c}$ \\
\hline
\end{tabular}

Table 3. The complexity of P-ABD, where *-subscripts on clones denote all valid completions, L abbreviates LOGSPACE, and the suffix "cc." indicates completeness for the respective complexity class.

Our results show, for instance, that when the knowledge base's formulae are restricted to be represented as positive clauses (i.e., $[B]=\mathrm{V}$ ), then the abduction problem for single literal manifestations is very easy (solvable in LOGSPACE); this still holds if the manifestations are represented by positive clauses. But its complexity jumps to NP-completeness if we change the restriction on the manifestations to allow for positive terms.

Considering the case that all monotonic functions can be simulated (i.e., $X \subseteq[B] \subseteq \mathrm{M}$ for $X \in\left\{\mathrm{D}_{2}, \mathrm{~S}_{00}, \mathrm{~S}_{10}\right\}$ ), the abduction problem is NP-complete for manifestations represented by literals, clauses, or terms. Here allowing manifestation represented by a monotonic formulae, causes the jump to $\Sigma_{2}^{\mathrm{p}}$-completeness. This increase in the complexity of the problem can be intuitively explained as follows. The complexity of the abduction rests on two sources: finding a candidate explanation and checking that it is indeed a solution. The NP-complete cases that occur in our classification hold for problems in which the verification can be performed in polynomial time. If both the knowledge base and the manifestation are represented by monotonic formulae, verifying a candidate explanation is coNP-complete.

It comes as no surprise that the complexity of $\mathrm{P}-\mathrm{ABD}(B, \mathcal{M})$ is lower than or equal to the complexity of $\operatorname{ABD}(B, \mathcal{M})$ in most cases (except for the affine clones). 
We have seen in Lemma 5.1 that for monotonic or 1-reproducing knowledge bases only one candidate needs to be considered. In these cases the complexity of the abduction problem is determined by the verification of the candidate. This explains the appearance of coNP-complete cases in our classification. For the affine clones (i.e., $[B] \in\left\{\mathrm{L}, \mathrm{L}_{0}, \mathrm{~L}_{3}\right\}$ ), on the other hand, the tractability of $\operatorname{ABD}(B, \mathcal{M})$ relies on Gaussian elimination. This method fails when restricting the hypotheses to be positive, and there is no obvious alternative.

\section{Counting complexity}

We now turn to the complexity of counting. We focus on the case where the manifestation is a single positive literal. For the symmetric abduction problem we are interested in counting the number of full explanations, \# $\mathrm{ABD}(B, \mathrm{PQ})$. For positive abduction two counting problems commonly arise: either to count all positive explanations, denoted by \# $\mathrm{P}-\mathrm{ABD}(B, \mathrm{PQ})$; or to count only the subsetminimal explanations, denoted by \#- $\subseteq-\mathrm{P}-\mathrm{ABD}(B, \mathrm{PQ})$. Our first result in this section is the complete classification of $\# \operatorname{ABD}(B, P Q)$.

Theorem 7.1. Let $B$ be a finite set of Boolean functions. Then the counting problem of symmetric abduction for propositional $B$-formulae with a positive literal manifestation, \# $\mathrm{ABD}(B, \mathrm{PQ})$, is

1. \#-coNP-complete if $\mathrm{S}_{02} \subseteq[B]$ or $\mathrm{S}_{12} \subseteq[B]$ or $\mathrm{D}_{1} \subseteq[B]$,

2. \#P-complete if $\mathrm{V}_{2} \subseteq[B] \subseteq \mathrm{M}$ or $\mathrm{S}_{10} \subseteq[B] \subseteq \mathrm{M}$ or $\mathrm{D}_{2} \subseteq[B] \subseteq \mathrm{M}$,

3. in $\mathrm{FP}$ in all other cases.

Proof. The \#·coNP-membership for \#ABD(B, PQ) follows from the facts that checking whether a set of literals is indeed an explanation for an abduction problem is in $\mathrm{P}^{\mathrm{NP}}=\Delta_{2}^{\mathrm{p}}$ and from the equality $\# \cdot \Delta_{2}^{\mathrm{p}}=\# \cdot \operatorname{coNP}$, see [HV95].

We show \#-coNP-hardness by giving a parsimonious reduction from the following \#·coNP-complete problem: Count the number of satisfying assignments of $\psi\left(x_{1}, \ldots, x_{n}\right)=\forall y_{1} \cdots \forall y_{m} \varphi\left(x_{1}, \ldots, x_{n}, y_{1}, \ldots, y_{m}\right)$, where $\varphi$ is a DNF-formula (see, e.g., [DHK05]). Let $x_{1}^{\prime}, \ldots, x_{n}^{\prime}, r_{1}, \ldots, r_{n}, t$ and $q$ be fresh, pairwise distinct propositions. We define the propositional abduction problem $\mathcal{P}=(\Gamma, A, q)$ as follows:

$$
\begin{aligned}
\Gamma: & =\left\{x_{i} \rightarrow r_{i}, x_{i}^{\prime} \rightarrow r_{i}, \neg x_{i} \vee \neg x_{i}^{\prime} \mid 1 \leq i \leq n\right\} \\
& \cup\{\varphi \rightarrow t\} \cup\left\{\bigwedge_{i=1}^{n} r_{i} \wedge t \rightarrow q\right\}, \\
A: & :=\left\{x_{1}, \ldots, x_{n}\right\} \cup\left\{x_{1}^{\prime}, \ldots, x_{n}^{\prime}\right\} .
\end{aligned}
$$

Observe that the manifestation $q$ occurs only in the formula $\bigwedge_{i=1}^{n} r_{i} \wedge t \rightarrow q$. This together with the formulae $x_{i} \rightarrow r_{i}, x_{i}^{\prime} \rightarrow r_{i}, \neg x_{i} \vee \neg x_{i}^{\prime}, 1 \leq i \leq n$, enforces that every full explanation of $\mathcal{P}$ has to select for each $i$ either $x_{i}$ and $\neg x_{i}^{\prime}$, or $\neg x_{i}$ and $x_{i}^{\prime}$. By this the value of $x_{i}^{\prime}$ is fully determined by the value of $x_{i}$ and is its dual. Moreover, it is easy to see that there is a one-to-one correspondence between the models of $\psi$ and the full explanations of $\mathcal{P}$. 
Observe that since the reductions in Lemma 3.1 are parsimonious, we can suppose w.l.o.g. that $B$ contains the two constants 1 and 0 . Therefore, it suffices to consider the case $[B]=\mathrm{BF}$. Suppose that $\varphi=\bigvee_{i \in I} t_{i}$ and let $\Gamma^{\prime}$ be the set of formulae obtained by replacing $\varphi \rightarrow t \equiv \neg\left(\bigvee_{i \in I} t_{i}\right) \vee t$ by the set of clauses $\left\{\neg t_{i} \vee t \mid i \in I\right\}$. Then $\Gamma^{\prime}$ is a set of disjunctions of literals, whose size is polynomially bounded by $|\Gamma|$. Hence, by the associativity of $\vee, \Gamma^{\prime}$ can be transformed in logarithmic space into an equivalent set of $B$-formulae. This provides a parsimonious reduction from the above \#-coNP-complete problem to \# $\mathrm{ABD}(B, \mathrm{PQ})$.

Let us now consider the \# $\mathrm{P}$-complete cases. When $[B] \subseteq \mathrm{M}$, checking whether a set of literals $E$ is an explanation for an abduction problem with $B$-formulae is in $\mathrm{P}$ (see Proposition 4.5). This proves membership in \#P. For the hardness result, it suffices to consider the case $[B]=\mathrm{V}_{2}$, because the reduction provided in Lemma 3.1 is parsimonious and $\mathrm{V}_{2} \subseteq\left[\mathrm{S}_{10} \cup\{1\}\right]$. We provide a Turing reduction from the problem \#Positive-2-SAT, which is known to be \#P-complete [Val79]. Let $\varphi=\bigwedge_{i=1}^{k}\left(p_{i} \vee q_{i}\right)$ be an instance of this problem, where $p_{i}$ and $q_{i}$ are propositional variables from the set $X=\left\{x_{1}, \ldots, x_{n}\right\}$. Let $q$ be a fresh proposition. Define the propositional abduction problem $\mathcal{P}=(\Gamma, A, q)$ as follows:

$$
\Gamma:=\left\{p_{i} \vee q_{i} \vee q \mid 1 \leq i \leq k\right\}, \quad A:=\left\{x_{1}, \ldots, x_{n}\right\} .
$$

It is easy to check that the number of satisfying assignments for $\varphi$ is equal to $2^{n}-\# \operatorname{Sol}(\mathcal{P})$. Finally, since $\vee \in[B]=\mathrm{V}_{2}, \mathcal{P}$ can easily be transformed in logarithmic space into an $\operatorname{ABD}(B, P Q)$-instance.

As for the tractable cases, the clones $\mathrm{E}$ and $\mathrm{N}$ are easy; and finally, for $[B] \subseteq \mathrm{L}$, the number of full explanations is polynomial time computable according to [HP07, Theorem 8].

Turning to positive abduction the \#P-complete cases vanish, while for the L-clones the exact complexity remains open.

Theorem 7.2. Let $B$ be a finite set of Boolean functions. Then both counting problems of positive abduction for propositional $B$-formulae with a positive literal manifestation, \# $\mathrm{P}-\mathrm{ABD}(B, \mathrm{PQ})$, \#-C-P-ABD $(B, \mathrm{PQ})$ are

1. \#·coNP-complete if $\mathrm{S}_{02} \subseteq[B]$ or $\mathrm{S}_{12} \subseteq[B]$ or $\mathrm{D}_{1} \subseteq[B]$,

2. in $\# \mathrm{P}$ if $\mathrm{L}_{2} \subseteq B \subseteq \mathrm{L}$,

3. in $\mathrm{FP}$ in all other cases.

Proof. The \#·coNP-membership follows analogously to the proof of Theorem 7.1. Indeed, the same reduction as in the proof of Theorem 7.1 works: there is an one-to-one correspondence between full explanations and purely positive explanations. Moreover, all explanations are incomparable and hence subset-minimal.

For the affine case membership in \#P follows from the NP-membership of the corresponding decision problem.

The remaining cases are encompassed by $[B] \subseteq \mathrm{M}$. In this case a slight strengthening of Lemma 5.1 is easily seen: Let $(\Gamma, A, q)$ be an instance. Then 
$A$ is an explanation if and only if all subsets of $A$ are explanations. Hence for \#P-ABD $(B, \mathrm{PQ})$ the number of solutions is either 0 or $2^{|A|}$ (all subsets), while for \#- $\subseteq-\mathrm{P}-\mathrm{ABD}(B, \mathrm{PQ})$ it is either 0 or 1 (the empty set). We obtain membership in FP, because for monotonic formulae deciding whether $A$ is an explanation can be done in LOGSPACE.

We note that for manifestations represented as terms, clauses, or $B$-formulae, most of the classifications of the corresponding counting problems can be easily derived from the above results; the exceptions to this are \# $\mathrm{ABD}(\mathrm{M}, \mathcal{L}(B))$ and some cases satisfying $[B \cup\{1\}]=\mathrm{L}$, whose exact complexity remains open.

\section{Concluding Remarks}

In this paper we studied the decision and counting complexity of symmetric and positive propositional abduction from a knowledge base being represented as sets of $B$-formulae, for all possible finite sets $B$ of Boolean functions. We gave a detailed picture of the complexity of abduction in considering restrictions on both manifestations and hypotheses. Thus our results highlight the sources of intractability, identify fragments of lower complexity, and may help to identify candidates for parameters in the study of parameterized complexity of abduction.

Our restrictions on the hypotheses covered only the symmetric and the positive case. One can as well define negative abduction, where explanations consist of negative literals only; or non-symmetric abduction, where explanations are formed upon a given set of literals, which is not demanded to be closed under complement (in contrast to S-ABD). However, results not mentioned herein indicate that the classifications of these variants are easily seen to be identical to $\mathrm{S}-\mathrm{ABD}$ (except for the L-clones).

It is worth noticing that, with the exception of the clones between $L_{2}$ and $L$, whenever the abduction problem is tractable for some clone, it is trivial. In contrast, tractability for the clones between $L_{2}$ and $L$ relies on Gaussian elimination, which fails when we restrict explanations to be positive. Determining the complexity of positive abduction for the clone $L$ with manifestations represented by L-formulae might hence prove to be a challenging task (note that a similar case, the circumscriptive inference of an affine formula from a set of affine formulae, remained unclassified in [Tho09]).

\section{References}

[AFM02] J. Amilhastre, H. Fargier, and P. Marquis. Consistency restoration and explanations in dynamic CSPs. Artif. Intell., 135(1-2):199-234, 2002.

[BATJ89] T. Bylander, D. Allemang, M. C. Tanner, and J. R. Josephson. Some results concerning the computational complexity of abduction. In Proc. 1st KR, pages 44-54, 1989.

[BDHM92] G. Buntrock, C. Damm, U. Hertrampf, and C. Meinel. Structure and importance of logspace MOD-classes. Mathematical Systems Theory, 25:223-237, 1992. 
[BHSS06] M. Bauland, E. Hemaspaandra, H. Schnoor, and I. Schnoor. Generalized modal satisfiability. In Proc. 23rd STACS, volume 3884 of $L N C S$, pages 500-511, 2006.

[BL00] M. Bouzid and A. Ligeza. Temporal causal abduction. Constraints, 5(3):303-319, 2000.

[BMTV09a] O. Beyersdorff, A. Meier, M. Thomas, and H. Vollmer. The complexity of propositional implication. Information Processing Letters, 109(18):10711077, 2009.

[BMTV09b] O. Beyersdorff, A. Meier, M. Thomas, and H. Vollmer. The complexity of reasoning for fragments of default logic. In Proc. 12th SAT, volume 5584 of $L N C S$, pages 51-64, 2009.

$\left[\mathrm{BSS}^{+} 08\right] \quad$ M. Bauland, T. Schneider, H. Schnoor, I. Schnoor, and H. Vollmer. The complexity of generalized satisfiability for linear temporal logic. In $L o g$ ical Methods in Computer Science, volume 5, 2008.

[CZ06] N. Creignou and B. Zanuttini. A complete classification of the complexity of propositional abduction. SIAM J. Comput., 36(1):207-229, 2006.

[DHK05] Arnaud Durand, Miki Hermann, and Phokion G. Kolaitis. Subtractive reductions and complete problems for counting complexity classes. Theoretical Computer Science, 340(3):496-513, 2005.

[EG95] T. Eiter and G. Gottlob. The complexity of logic-based abduction. J. ACM, 42(1):3-42, 1995.

[HP07] M. Hermann and R. Pichler. Counting complexity of propositional abduction. In Proc. 20th IJCAI, pages 417-422, 2007.

[HSAM93] J. R. Hobbs, M. E. Stickel, D. E. Appelt, and P. A. Martin. Interpretation as abduction. Artif. Intell., 63(1-2):69-142, 1993.

[HV95] L. Hemaspaandra and H. Vollmer. The satanic notations: counting classes beyond \#P and other definitional adventures. Complexity Theory Column 8, ACM-SIGACT News, 26(1):2-13, 1995.

[Lew79] H. Lewis. Satisfiability problems for propositional calculi. Mathematical Systems Theory, 13:45-53, 1979.

[NZ05] G. Nordh and B. Zanuttini. Propositional abduction is almost always hard. In Proc. 19th IJCAI, pages 534-539, 2005.

[NZ08] G. Nordh and B. Zanuttini. What makes propositional abduction tractable. Artif. Intell., 172(10):1245-1284, 2008.

[Pap94] C. H. Papadimitriou. Computational Complexity. Addison-Wesley, 1994.

[Pos41] E. Post. The two-valued iterative systems of mathematical logic. Annals of Mathematical Studies, 5:1-122, 1941.

[Rei03] S. Reith. On the complexity of some equivalence problems for propositional calculi. In Proc. 28th MFCS, volume 2747 of LNCS, pages 632-641, 2003.

[Sch78] T. J. Schaefer. The complexity of satisfiability problems. In Proc. 10th STOC, pages 216-226, 1978.

[Sch05] H. Schnoor. The complexity of the Boolean formula value problem. Technical report, Theoretical Computer Science, University of Hannover, 2005.

[SL90] B. Selman and H. Levesque. Abductive and default reasoning: A computational core. In Proc. 8th AAAI, pages 343-348, 1990.

[SW01] M. Stumptner and F. Wotawa. Diagnosing tree-structured systems. Artif. Intell., 127(1):1-29, 2001. 
[Tho09] M. Thomas. The complexity of circumscriptive inference in Post's lattice. In Proc. 10th LPNMR, volume 5753 of Lecture Notes in Computer Science, pages 290-302, 2009.

[Val79] L. G. Valiant. The complexity of enumeration and reliability problems. SIAM J. Comput., 8(3):411-421, 1979.

[Vol09] H. Vollmer. The complexity of deciding if a boolean function can be computed by circuits over a restricted basis. Theory of Computing Systems, 44(1):82-90, July 2009.

[Wra77] C. Wrathall. Complete sets and the polynomial-time hierarchy. Theoretical Computer Science, 3:23-33, 1977.

[Zan03] B. Zanuttini. New polynomial classes for logic-based abduction. J. Artif. Intell. Res., 19:1-10, 2003. 


\begin{tabular}{|c|c|c|}
\hline Name & Definition & Base \\
\hline $\mathrm{BF}$ & All Boolean functions & $\{x \wedge y, \neg x\}$ \\
\hline$\overline{\mathrm{R}_{0}}$ & $\{f \mid f$ is 0-reproducing $\}$ & $\{x \wedge y, x \oplus y\}$ \\
\hline $\mathrm{R}_{1}$ & $\{f \mid f$ is 1-reproducing $\}$ & $\{x \vee y, x \oplus y \oplus 1\}$ \\
\hline $\mathrm{R}_{2}$ & $\mathrm{R}_{0} \cap \mathrm{R}_{1}$ & $\{\vee, x \wedge(y \oplus z \oplus 1)\}$ \\
\hline $\bar{M}$ & $\{f \mid f$ is monotonic $\}$ & $\{x \vee y, x \wedge y, 0,1\}$ \\
\hline $\mathrm{M}_{1}$ & $\mathrm{M} \cap \mathrm{R}_{1}$ & $\{x \vee y, x \wedge y, 1\}$ \\
\hline $\mathrm{M}_{0}$ & $\mathrm{M} \cap \mathrm{R}_{0}$ & $\{x \vee y, x \wedge y, 0\}$ \\
\hline$\overline{\mathrm{M}_{2}}$ & $\mathrm{M} \cap \mathrm{R}_{2}$ & $\{x \vee y, x \wedge y\}$ \\
\hline $\mathrm{S}_{0}^{n}$ & $\{f \mid f$ is 0-separating of degree $n\}$ & $\left\{x \rightarrow y, \operatorname{dual}\left(h_{n}\right)\right\}$ \\
\hline $\mathrm{S}_{0}$ & $\{f \mid f$ is 0 -separating $\}$ & $\{x \rightarrow y\}$ \\
\hline$\overline{\mathrm{S}_{1}^{n}}$ & $\{f \mid f$ is 1-separating of degree $n\}$ & $\left\{x \wedge \neg y, h_{n}\right\}$ \\
\hline $\mathrm{S}_{1}$ & $\{f \mid f$ is 1-separating $\}$ & $\{x \wedge \neg y\}$ \\
\hline$\overline{\mathrm{S}_{02}^{n}}$ & $\mathrm{~S}_{0}^{n} \cap \mathrm{R}_{2}$ & $\left\{x \vee(y \wedge \neg z), \operatorname{dual}\left(h_{n}\right)\right\}$ \\
\hline $\mathrm{S}_{02}$ & $\mathrm{~S}_{0} \cap \mathrm{R}_{2}$ & $\{x \vee(y \wedge \neg z)\}$ \\
\hline $\mathrm{S}_{01}^{n}$ & $\mathrm{~S}_{0}^{n} \cap \mathrm{M}$ & $\left\{\operatorname{dual}\left(h_{n}\right), 1\right\}$ \\
\hline $\mathrm{S}_{01}$ & $\mathrm{~S}_{0} \cap \mathrm{M}$ & $\{x \vee(y \wedge z), 1\}$ \\
\hline$\overline{\mathrm{S}_{00}^{n}}$ & $\mathrm{~S}_{0}^{n} \cap \mathrm{R}_{2} \cap \mathrm{M}$ & $\left\{x \vee(y \wedge z), \operatorname{dual}\left(h_{n}\right)\right\}$ \\
\hline $\mathrm{S}_{00}$ & $\mathrm{~S}_{0} \cap \mathrm{R}_{2} \cap \mathrm{M}$ & $\{x \vee(y \wedge z)\}$ \\
\hline $\mathrm{S}_{12}^{n}$ & $\mathrm{~S}_{1}^{n} \cap \mathrm{R}_{2}$ & $\left\{x \wedge(y \vee \neg z), h_{n}\right\}$ \\
\hline$\overline{\mathrm{S}_{12}}$ & $\mathrm{~S}_{1} \cap \mathrm{R}_{2}$ & $\{x \wedge(y \vee \neg z)\}$ \\
\hline $\mathrm{S}_{11}^{n}$ & $\mathrm{~S}_{1}^{n} \cap \mathrm{M}$ & $\left\{h_{n}, 0\right\}$ \\
\hline $\mathrm{S}_{11}$ & $\mathrm{~S}_{1} \cap \mathrm{M}$ & $\{x \wedge(y \vee z), 0\}$ \\
\hline $\mathrm{S}_{10}^{n}$ & $\mathrm{~S}_{1}^{n} \cap \mathrm{R}_{2} \cap \mathrm{M}$ & $\left\{x \wedge(y \vee z), h_{n}\right\}$ \\
\hline $\mathrm{S}_{10}$ & $\mathrm{~S}_{1} \cap \mathrm{R}_{2} \cap \mathrm{M}$ & $\{x \wedge(y \vee z)\}$ \\
\hline$\overline{\mathrm{D}}$ & $\{f \mid f$ is self-dual $\}$ & $\{(x \wedge \neg y) \vee(x \wedge \neg z) \vee(\neg y \wedge \neg z)\}$ \\
\hline$\overline{D_{1}}$ & $\mathrm{D} \cap \mathrm{R}_{2}$ & $\{(x \wedge y) \vee(x \wedge \neg z) \vee(y \wedge \neg z)\}$ \\
\hline$\overline{\mathrm{D}_{2}}$ & $\mathrm{D} \cap \mathrm{M}$ & $\{(x \wedge y) \vee(x \wedge z) \vee(y \wedge z)\}$ \\
\hline $\mathrm{L}$ & $\{f \mid f$ is affine $\}$ & $\{x \oplus y, 1\}$ \\
\hline $\mathrm{L}_{0}$ & $\mathrm{~L} \cap \mathrm{R}_{0}$ & $\{x \oplus y\}$ \\
\hline $\mathrm{L}_{1}$ & $\mathrm{~L} \cap \mathrm{R}_{1}$ & $\{x \oplus y \oplus 1\}$ \\
\hline $\mathrm{L}_{2}$ & $\mathrm{~L} \cap \mathrm{R}_{2}$ & $\{x \oplus y \oplus z\}$ \\
\hline $\mathrm{L}_{3}$ & $\mathrm{~L} \cap \mathrm{D}$ & $\{x \oplus y \oplus z \oplus 1\}$ \\
\hline$\overline{\mathrm{V}}$ & $\{f \mid f$ is a disjunction of variables or constants $\}$ & $\{x \vee y, 0,1\}$ \\
\hline$\overline{\mathrm{V}_{0}}$ & $\mathrm{~V} \cap \mathrm{R}_{0}$ & $\{x \vee y, 0\}$ \\
\hline$\overline{V_{1}}$ & $\mathrm{~V} \cap \mathrm{R}_{1}$ & $\{x \vee y, 1\}$ \\
\hline $\mathrm{V}_{2}$ & $\mathrm{~V} \cap \mathrm{R}_{2}$ & $\{x \vee y\}$ \\
\hline $\mathrm{E}$ & $\{f \mid f$ is a conjunction of variables or constants $\}$ & $\{x \wedge y, 0,1\}$ \\
\hline $\mathrm{E}_{0}$ & $\mathrm{E} \cap \mathrm{R}_{0}$ & $\{x \wedge y, 0\}$ \\
\hline $\mathrm{E}_{1}$ & $\mathrm{E} \cap \mathrm{R}_{1}$ & $\{x \wedge y, 1\}$ \\
\hline $\mathrm{E}_{2}$ & $\mathrm{E} \cap \mathrm{R}_{2}$ & $\{x \wedge y\}$ \\
\hline $\mathrm{N}$ & $\{f \mid f$ depends on at most one variable $\}$ & $\{\neg x, 0,1\}$ \\
\hline $\mathrm{N}_{2}$ & $\mathrm{~N} \cap \mathrm{R}_{2}$ & $\{\neg x\}$ \\
\hline I & $\{f \mid f$ is a projection or a constant $\}$ & $\{\mathrm{id}, 0,1\}$ \\
\hline $\mathrm{I}_{0}$ & $I \cap R_{0}$ & $\{\mathrm{id}, 0\}$ \\
\hline$I_{1}$ & $\mathrm{I} \cap \mathrm{R}_{1}$ & $\{$ id, 1$\}$ \\
\hline $\mathrm{I}_{2}$ & $\mathrm{I} \cap \mathrm{R}_{2}$ & $\{$ id $\}$ \\
\hline
\end{tabular}

Table 1. The list of all Boolean clones with definitions and bases, where $h_{n}:=$ $\bigvee_{i=1}^{n+1} \bigwedge_{j=1, j \neq i}^{n+1} x_{j}$ and $\operatorname{dual}(f)\left(a_{1}, \ldots, a_{n}\right)=\neg f\left(\neg a_{1} \ldots, \neg a_{n}\right)$. 


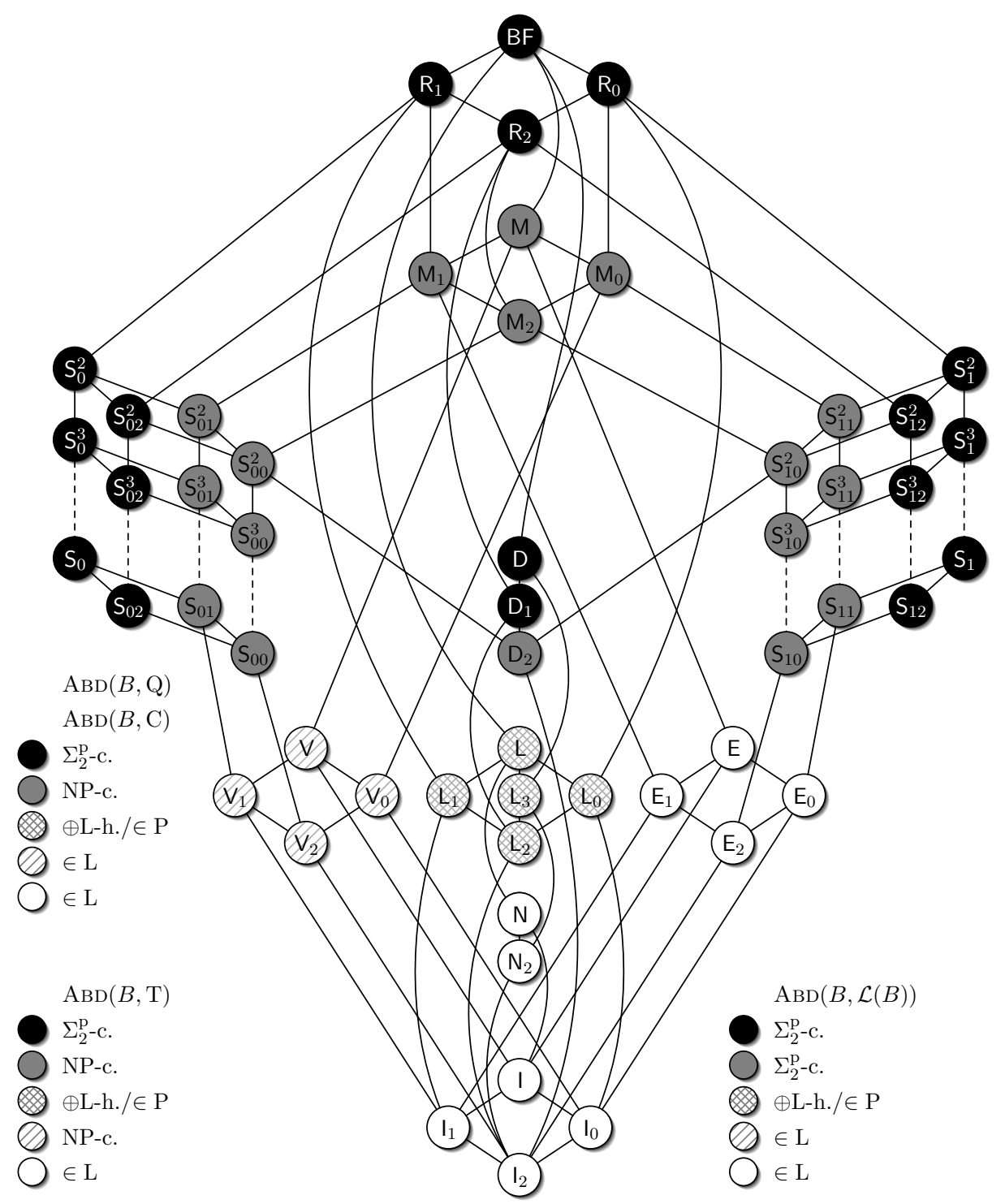

Fig. 1. Post's lattice showing the complexity of the symmetric abduction problem $\operatorname{ABD}(B, \mathcal{M})$ for all sets $B$ of Boolean functions and the most interesting restrictions $\mathcal{M}$ of the manifestations. In the legend, L abbreviates LOGSPACE and the suffixes "-h" and "-c" indicate hardness and completeness respectively. 


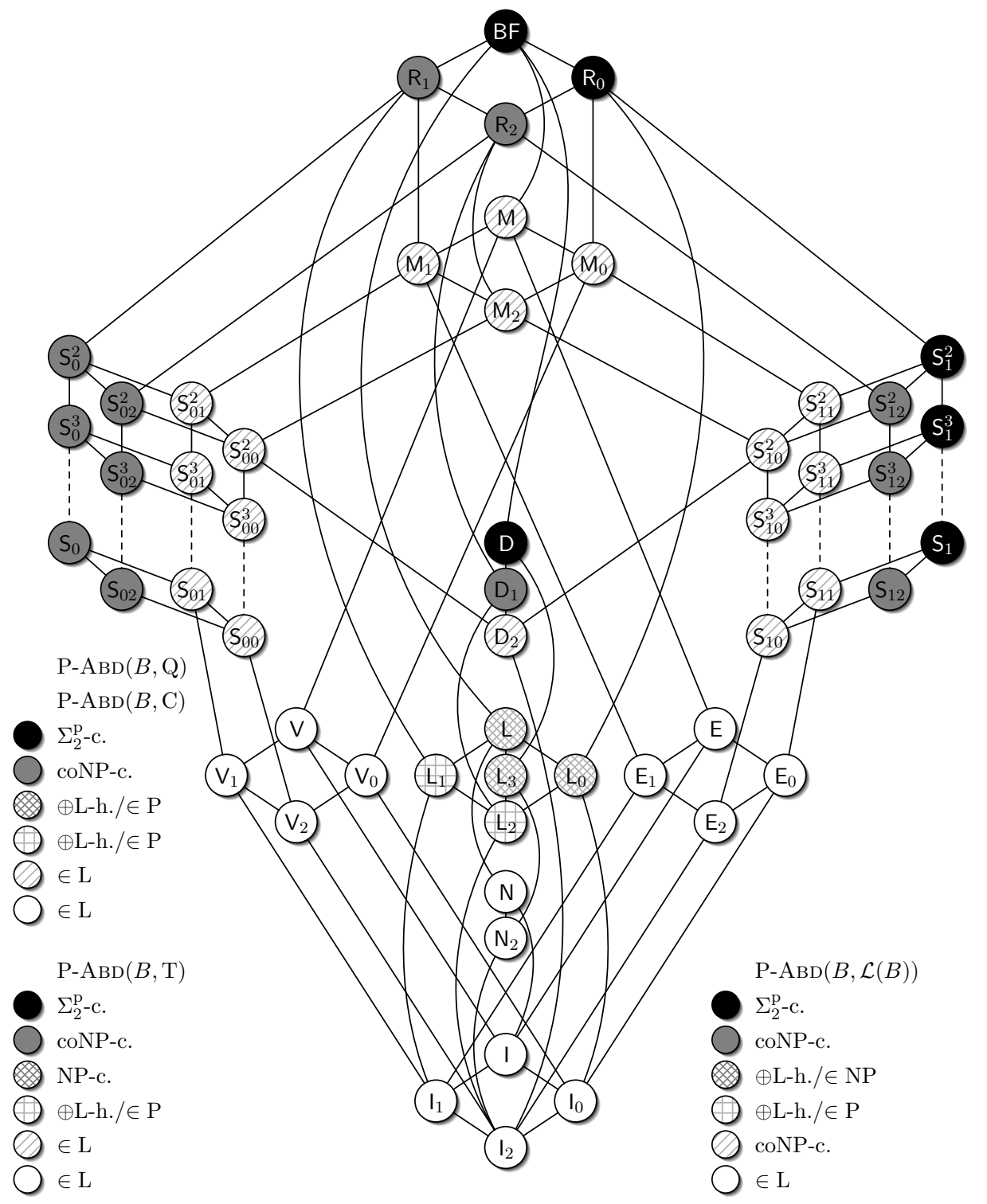

Fig. 2. Post's lattice showing the complexity of the positive abduction problem $\operatorname{P}-\operatorname{ABD}(B, \mathcal{M})$ for all sets $B$ of Boolean functions and the most interesting restrictions $\mathcal{M}$ of the manifestations. In the legend, L abbreviates LOGSPACE and the suffixes "-h" and "-c" indicate hardness and completeness respectively. 